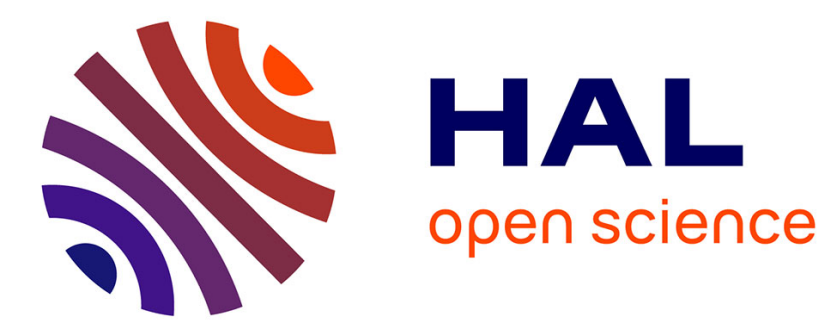

\title{
Singular mean-field control games
}

\author{
Yaozhong Hu, Bernt Øksendal, Agnès Sulem
}

\section{To cite this version:}

Yaozhong Hu, Bernt Øksendal, Agnès Sulem. Singular mean-field control games. Stochastic Analysis and Applications, 2017, 35 (5), pp.823-851. 10.1080/07362994.2017.1325745 . hal-01614747

\section{HAL Id: hal-01614747 \\ https://hal.inria.fr/hal-01614747}

Submitted on 18 Oct 2017

HAL is a multi-disciplinary open access archive for the deposit and dissemination of scientific research documents, whether they are published or not. The documents may come from teaching and research institutions in France or abroad, or from public or private research centers.
L'archive ouverte pluridisciplinaire HAL, est destinée au dépôt et à la diffusion de documents scientifiques de niveau recherche, publiés ou non, émanant des établissements d'enseignement et de recherche français ou étrangers, des laboratoires publics ou privés. 


\title{
Singular mean-field control games
}

\author{
Yaozhong $\mathrm{Hu}^{1,2}$, Bernt $\varnothing \mathrm{ksendal^{2,3 }}$, Agnès Sulem ${ }^{4}$ \\ 28 April 2017
}

MSC(2010): 60H10, 91A15, 91A23, 91B38, 91B55, 91B70, 93E20

Keywords: Optimal singular control; Mean-field stochastic differential equations; Brownian motion, Poisson random measures; Singular mean-field control games; Stochastic maximum principles; Skorohod reflection problem; Nash equilibrium.

\begin{abstract}
This paper studies singular mean field control problems and singular mean field twoplayers stochastic differential games. Both sufficient and necessary conditions for the optimal controls and for the Nash equilibrium are obtained. Under some assumptions the optimality conditions for singular mean-field control are reduced to a reflected Skorohod problem, whose solution is proved to exist uniquely. Motivations are given as optimal harvesting of stochastic mean-field systems, optimal irreversible investments under uncertainty and mean-field singular investment games. In particular, a simple singular mean-field investment game is studied, where the Nash equilibrium exists but is not unique.
\end{abstract}

\section{Introduction}

Mean-field stochastic differential equations (SDEs) have been the subject of much attention in the mathematics research literature recently. This is mainly due to their interesting applications in mathematical physics and more recently in models for systemic risk, but also because they represent natural generalizations of independent interest of classical SDEs.

${ }^{1}$ Department of Mathematics, University of Kansas, Lawrence, Kansas 66045, USA. Email: yhu@ku.edu. $\mathrm{Y}$. Hu is partially supported by a grant from the Simons Foundation \#209206 and by a General Research Fund of University of Kansas.

${ }^{2}$ Department of Mathematics, University of Oslo, P.O. Box 1053 Blindern, N-0316 Oslo, Norway. Email: oksendal@math.uio.no.

${ }^{3}$ This research was carried out with support of the Norwegian Research Council, within the research project Challenges in Stochastic Control, Information and Applications (STOCONINF), project number 250768/F20.

${ }^{4}$ INRIA Paris, 3 rue Simone Iff, CS 42112, 75589 Paris Cedex 12, France; Email: agnes.sulem@inria.fr 
The purpose of this paper is to study singular control problems for mean-field SDEs, and to give methods for solving such problems, in terms of finding the optimal controls. We shall also study in this paper singular mean-field non-zero stochastic differential games. Let us point out that the terminology "mean field game" has been used in the literature to represent the limit when the number of players in the game becomes large (see e.g. [12], [14], [6]). Here we consider two-players games with mean-field terms in the state dynamics and in the performance functional. We establish necessary and sufficient maximum principles for the optimal control for such games and their corresponding Nash equilibria. To illustrate the theory we give some solvable examples and provide cases where the Nash equilibrium is not unique. See e.g. Proposition 5.1.

For mean field singular control the stochastic maximum principle method yields backward stochastic differential equations with mean field term and with reflection. We shall combine here the contraction mapping technique with some recent results of Burdzy, Kang and Ramanan (2009) on the Skorohod mapping for time dependent interval [4] to obtain the existence and uniqueness of these equations. Note that we assume local convexity of the set of admissible controls to avoid second order adjoint equations for the necessary maximum principle (see [23], [25]). We assume some concavity conditions of the Hamiltonian and terminal payoff to get sufficient optimality conditions (see Theorem 3.2 and Theorem 4.1 below).

Recently, there have been several works dealing with mean-field control problems. See e.g. Bensoussan et al [2], Buckdahn et al (2011) [3], Carmona and Delarue (2013) [5], MeyerBrandis et al (2012) [15], Anderson and Djehiche (2011) [1] and Hamadène (1998) [9].

The article most closely related to ours is a recent paper [26] by L. Zhang (2012), dealing with mean-field singular control. Our paper extends this paper in several directions: First, the mean-field operators are extended to a general (nonlinear) Fréchet differentiable functional $Y$ of the state variable. Secondly, we allow the profit rate $f$ in the performance functional to depend on the mean-field term $Y$ as well as the singular control $\xi$, in addition to its dependence on the state $X$ itself. Third, we allow both the coefficient $\lambda$ in the singular part of the state equation and the singular cost coefficient $h$ in the performance functional to depend on the state $X$. Moreover, we consider games between two players of such singular control problems with asymmetric information. Let us also mention that in our paper's framework, but without singular control, a stochastic maximum principle is obtained and the existence of Nash equilibria is proved in [10].

This paper is organized as follows: In Section 2 we present three motivating examples. In Section 3 we formulate a general mean-field singular stochastic control problem and prove a sufficient maximum principle and a necessary maximum principle. We then reduce the maximum principle to a Skorohod problem and prove the existence and uniqueness of the solution. We illustrate our results on an optimal harvesting problem of a mean-field system. In Section 4 we prove maximum principles for singular mean-field stochastic games with two players and we obtain as a corollary a corresponding maximum principle for zero-sum games. In Section 5 we apply these results to a mean field investment game and to singular control with model uncertainty. 


\section{Three motivating examples}

\subsection{Optimal harvesting from a mean-field system}

Let us model the density $X^{0}(t)$ of an unharvested population at time $t$ by an equation of the form

$$
d X^{0}(t)=\mathbb{E}\left[X^{0}(t)\right] b(t) d t+X^{0}(t) \sigma(t) d B(t), \quad t \in[0, T] ; \quad X^{0}(0)=x>0 .
$$

Here, and in the following, $B(t)=B(t, \omega)$ is a Brownian motion on a filtered probability space $\left(\Omega, \mathcal{F}, \mathbb{F}:=\left\{\mathcal{F}_{t}\right\}_{t \geq 0}, P\right)$ satisfying the usual conditions. $P$ is a reference probability measure, and we assume that $\mathbb{F}$ is the Brownian filtration. We assume that $b(t)$ and $\sigma(t)$ are given predictable processes. We may heuristically regard (2.1) as a limit as $n \rightarrow \infty$ of a large population interacting system of the form

$$
d x^{i, n}(t)=\left[\frac{1}{n} \sum_{j=1}^{n} x^{j, n}(t)\right] b(t) d t+x^{i, n}(t) \sigma(t) d B^{i}(t), \quad i=1,2, \ldots, n
$$

where we have devided the whole lake into a grid of size $n$ and $x^{i, n}(t)$ represents the density in box $i$ of the grid. Thus the mean-field term $\mathbb{E}[X(t)]$ represents an approximation to the weighted average $\frac{1}{n} \sum_{j=1}^{n} x^{j, n}(t)$ for large $n$.

Now suppose we introduce harvesting of the population. The density of the harvested population $X(t)=X^{\xi}(t)$ at time $t$ can then be modeled by a mean-field singular control stochastic differential equation of the form

$$
d X(t)=\mathbb{E}[X(t)] b(t) d t+X(t) \sigma(t) d B(t)-\lambda_{0}(t) d \xi(t) ; \quad t \in[0, T] ; X(0)=x>0
$$

where $\xi(t)$ is a non-decreasing predictable process with $\xi\left(0^{-}\right)=0$, representing the harvesting effort, while $\lambda_{0}(t)>0$ is a given harvesting efficiency coefficient.

The performance functional is assumed to be of the form

$$
J(\xi)=\mathbb{E}\left[\int_{o}^{T} h_{0}(t) X(t) d \xi(t)+K X(T)\right],
$$

where $h_{0}(t)$ is a given adapted price process and $K=K(\omega)$ is a given salvage price, assumed to be $\mathcal{F}_{T}$-measurable. The problem is to find $\xi^{*}$ such that

$$
J\left(\xi^{*}\right)=\sup _{\xi} J(\xi)
$$

Such a process $\xi^{*}$ is called an optimal singular control. This is an example of a mean-field singular control problem. We will return to this problem in Example 3.5. 


\subsection{A mean field investment game}

Arguing as in Section 2.1 let us assume that the demand density (demand per area unit) $X(t)$ for a certain product at time $t$ is given by a mean field SDE of the form

$$
d X(t)=\mathbb{E}[X(t)] b(t) d t+X(t) \sigma(t) d B(t) ; X(0)=x>0 .
$$

Alternatively, we might think of $X(t)$ as the demand at time $t$ of a representative agent. There are two competing companies producing this product, with production rate capacities represented by nondecreasing adapted processes $\xi_{1}, \xi_{2}$, respectively. The expected profit of the company $i$ is assumed to have the form

$$
J_{i}\left(\xi_{1}, \xi_{2}\right)=\mathbb{E}\left[\int_{0}^{T} \pi(t) \min \left(X(t), \xi_{1}(t)+\xi_{2}(t)\right) d t+\int_{0}^{T} h_{i}(t) d \xi_{i}(t)\right],
$$

where $\pi(t)>0$ is the price per unit sold and $h_{i}(t)<0$ is the production cost per unit for the factory $i, i=1,2$. We want to find a Nash equilibrium, i.e. a pair $\left(\xi_{1}^{*}, \xi_{2}^{*}\right) \in \mathcal{A}_{1} \times \mathcal{A}_{2}$ such that

$$
\sup _{\xi_{1} \in \mathcal{A}_{1}} J_{1}\left(\xi_{1}, \xi_{2}^{*}\right) \leq J_{1}\left(\xi_{1}^{*}, \xi_{2}^{*}\right)
$$

and

$$
\sup _{\xi_{2} \in \mathcal{A}_{2}} J_{2}\left(\xi_{1}^{*}, \xi_{2}\right) \leq J_{2}\left(\xi_{1}^{*}, \xi_{2}^{*}\right),
$$

where $\mathcal{A}_{i}$ is the family of admissible controls $\xi_{i}$ for company number $i ; i=1,2$. We will return to this problem in Section 5.1.

\subsection{Optimal irreversible investments under model uncertainty}

The irreversible investment problem is a classical problem in economics, with a long history. It has been studied by many authors in different contexts. See e.g. Pindyck (1988, 1991, 1991),[20, 21, 22], Kobila (1993) [13] and the references therein.

In short, the problem is the following. A factory is facing an increased demand for its product. Should it invest in more production capacity to meet the demand? The problem is that buying additional production capacity is an expensive, irreversible investment (usually production equipment cannot easily be sold after use) and the future demand for the product is uncertain. So the risk is that the factory ends up having paid for an additional capacity it does not need. On the other hand, if the factory does not increase the capacity, it might miss an opportunity for an increased sale. Mathematically the problem can be formulated as a singular control problem: indeed, since the investment is irreversible, the control of the investor is a singular control, i.e. a non-decreasing non-negative stochastic process.

Let $\xi(t)$ denote the production rate capacity of a production plant and let $X(t)$ denote the demand rate at time $t$. At any time $t$ the production capacity can be increased by $d \xi(t)$ at the price $h(t, X(t))$ per capacity unit. The number of units sold per time unit is the 
minimum of the demand $X(t)$ and the capacity $\xi(t)$. The total expected net profit of the production is assumed to be

$$
J(\xi, \theta)=\mathbb{E}^{Q_{\theta}}\left[\int_{0}^{T} a(t, \mathbb{E}[\varphi(X(t))]) \min [X(t), \xi(t)] d t+g(X(T))-\int_{0}^{T} h(t, X(t)) d \xi(t)\right]
$$

where $g(X(T))$ is some salvage value of the closed-down production plant, $\mathbb{E}[\varphi(X(t))]$ denotes the expectation with respect to a reference probability measure $P, \varphi$ is a given real function and $a(t, \mathbb{E}[\varphi(X(t))])$ is the unit sales price of the production. We think it gives a more realistic model to allow the sales price to depend on the average demand rate of the product at any give time.

We study this problem under model uncertainty, in the sense of Knight uncertainty, i.e. uncertainty about the underlying probability measure. Using the Girsanov theorem for Itô processes we can parameterize the family of densities of possible underlying probability measures by a stochastic process $\theta(t)$.

Here $\left\{Q^{\theta}\right\}_{\theta \in \Theta}$ is a family of probability measures representing the model uncertainty. We let $\mathcal{A}_{\mathbb{G}}$ denote the set of right-continuous, non-decreasing $\mathbb{G}$-adapted processes $\xi(\cdot)$ with $\xi\left(0^{-}\right)=0$, where $\mathbb{G}:=\left\{\mathcal{G}_{t}\right\}_{t \geq 0}$ is a given subfiltration of $\mathbb{F}$, in the sense that $\mathcal{G}_{t} \subseteq \mathcal{F}_{t}$ for all $t$. Heuristically, $\mathcal{G}_{t}$ represents the information available to the investor at time $t$. We assume that the demand $X(t)$ is given by a diffusion of the form

$$
d X(t)=X(t)[\alpha(t, \omega) d t+\beta(t, \omega) d B(t)], 0 \leq t \leq T ; X(0)>0
$$

where $\alpha(t, \omega), \beta(t, \omega)$ are given $\mathbb{F}$-adapted processes. We want to maximize the expected total net profit under the worst possible scenario, i.e. find $\left(\xi^{*}, \theta^{*}\right) \in \mathcal{A}_{\mathbb{G}} \times \Theta$ such that

$$
\sup _{\xi \in \mathcal{A}_{\mathbb{G}}} \inf _{\theta \in \Theta} J(\xi, \theta)=\inf _{\theta \in \Theta} \sup _{\xi \in \mathcal{A}_{\mathbb{G}}} J(\xi, \theta)=J\left(\xi^{*}, \theta^{*}\right)
$$

This leads to a stochastic differential zero-sum game in which one of the players is the investor controlling the investment strategy and the other player controls the model parameter $\theta$.

This is an example of a (partial information) singular control game problem. Note that the system is non-Markovian, both because of the mean-field term and the partial information constraint. See Section 5.2.

\section{Maximum principles for singular mean field control problems}

\subsection{Problem statement}

We first recall some basic concepts and results from Banach space theory. Throughout this paper, we assume that the probability space $\Omega$ is a Banach space, where $(\Omega, \mathcal{F}, \mathbb{F}:=$ 
$\left.\left\{\mathcal{F}_{t}\right\}_{t \geq 0}, P\right)$ is as introduced in the beginning of Section 2. Throughout this paper $\mathbb{G}:=$ $\left\{\mathcal{G}_{t}\right\}_{t \geq 0}$ is a given subfiltration of $\mathbb{F}$.

Let $V$ be an open subset of a Banach space $\mathcal{X}$ with norm $\|\cdot\|$ and let $F: V \rightarrow \mathbb{R}$.

(i) We say that $F$ has a directional derivative (or Gâteaux derivative) at $x \in \mathcal{X}$ in the direction $y \in \mathcal{X}$ if

$$
D_{y} F(x):=\lim _{\varepsilon \rightarrow 0} \frac{1}{\varepsilon}(F(x+\varepsilon y)-F(x))
$$

exists.

(ii) We say that $F$ is Fréchet differentiable at $x \in V$ if there exists a continuous linear map $L: \mathcal{X} \rightarrow \mathbb{R}$ such that

$$
\lim _{\substack{h \rightarrow 0 \\ h \in \mathcal{X}}} \frac{1}{\|h\|}|F(x+h)-F(x)-L(h)|=0 .
$$

In this case we call $L$ the gradient (or Fréchet derivative) of $F$ at $x$ and we write

$$
L=\nabla_{x} F .
$$

(iii) If $F$ is Fréchet differentiable, then $F$ has a directional derivative in all directions $y \in \mathcal{X}$ and

$$
D_{y} F(x)=\nabla_{x} F(y)=:\left\langle\nabla_{x} F, y\right\rangle .
$$

In particular, if $\mathcal{X}=L^{2}(P)$ the Fréchet derivative of $F$ at $X \in L^{2}(P)$, denoted by $\nabla_{X} F$, is a bounded linear functional on $L^{2}(P)$. Since $L^{2}(P)$ is a Hilbert space, we can identify $\nabla_{x} F$ with a random variable in $L^{2}(P)$. We shall then regard $\nabla_{x} F$ as an element in $L^{2}$. For example, if $F(X)=E[\varphi(X)] ; X \in L^{2}(P)$, where $\varphi$ is a real $C^{1}$ - function such that $\varphi(X) \in$ $L^{2}(P)$ and $\frac{\partial \varphi}{\partial x}(X) \in L^{2}(P)$, then with the above identification, we have $\nabla_{X} F=\frac{\partial \varphi}{\partial x}(X)$ and $\nabla_{X} F(Y)=\left\langle\frac{\partial \varphi}{\partial x}(X), Y\right\rangle=E\left[\frac{\partial \varphi}{\partial x}(X) Y\right]$ for $Y \in L^{2}(P)$.

Consider a mixed regular and singular controlled finite dimensional system with state process $X(t)=X^{\xi, u}(t)$ of the form

$$
\begin{aligned}
d X(t) & =b(t, X(t), Y(t), \xi(t), u(t), \omega) d t+\sigma(t, X(t), Y(t), \xi(t), u(t), \omega) d B(t) \\
& +\lambda(t, X(t), u(t), \omega) d \xi(t),
\end{aligned}
$$

where $X(t) \in \mathbb{R}^{d}$ is a $d$ dimensional adapted process, $\xi(t)$ and $u(t)$ are $\mathbb{R}^{m}$ and $\mathbb{R}^{n}$ valued $\mathbb{G}$-adapted processes,

$$
Y(t)=F(X(t, \cdot))
$$

and $F$ is a Fréchet differentiable operator on $L^{2}(P)$. 
The performance functional is assumed to be of the form

$$
\begin{aligned}
J(\xi, u)=\mathbb{E} & {\left[\int_{0}^{T} f(t, X(t), Y(t), \xi(t), u(t), \omega) d t+g(X(T), Y(T), \omega)\right.} \\
& \left.+\int_{0}^{T} h(t, X(t), \xi(t), u(t), \omega) d \xi(t)\right] .
\end{aligned}
$$

We may interpret the function $f$ as a profit rate, $g$ as a bequest or salvage value function and $h$ as a cost rate for the use of the singular control $\xi$.

Assumption 3.1. Throughout this paper we assume that the functions $b, \sigma, \lambda, f, g$ and $h$ are progressively measurable with respect to $(t, \omega)$ for all fixed $x, y, \xi, u$ and continuously differentiable with respect to $x, y, \xi, u$ with bounded derivatives.

We want to find $\left(\xi^{*}, u^{*}\right) \in \mathcal{A}$ such that

$$
J\left(\xi^{*}, u^{*}\right)=\sup _{(\xi, u) \in \mathcal{A}} J(\xi, u) .
$$

Here $\mathcal{A}=\mathcal{A}_{\mathbb{G}}$ is the family of $\mathbb{G}$-predictable processes such that the corresponding state equation has a unique solution $X$ such that $\omega \rightarrow X(t, \omega) \in L^{2}(P)$ for all t. We let $A$ denote the set of possible values of $u(t) ; t \in[0, T]$ when $(\xi, u) \in \mathcal{A}$.

\subsection{A sufficient maximum principle}

We prove below a sufficient maximum principle for the singular control problem described by (3.1) and (3.3). To this end, define the singular Hamiltonian $H$ as follows:

$$
\begin{aligned}
& H(t, x, y, \xi, u, p, q)(d t, \xi(d t)) \\
& \quad=H_{0}(t, x, y, \xi, u, p, q) d t+\{\lambda(t, x, u) p+h(t, x, \xi, u)\} d \xi(t),
\end{aligned}
$$

where

$$
H_{0}(t, x, y, \xi, u, p, q):=f(t, x, y, \xi, u)+b(t, x, y, \xi, u) p+\sigma(t, x, y, \xi, u) q .
$$

We assume that for $H$ is Fréchet differentiable $\left(C^{1}\right)$ in the variables $x, y, \xi, u$.

The associated mean-field BSDE for the adjoint processes is

$$
\left\{\begin{aligned}
d p(t) & =-\frac{\partial H_{0}}{\partial x}(t, X(t), Y(t), \xi(t), u(t), p(t), q(t)) d t \\
& -\frac{\partial H_{0}}{\partial y}(t, X(t), Y(t), \xi(t), u(t), p(t), q(t)) \nabla_{X(t)} F d t \\
& -\left\{\frac{\partial \lambda}{\partial x}(t, X(t), u(t)) p(t)+\frac{\partial h}{\partial x}(t, X(t), u(t))\right\} d \xi(t)+q(t) d B(t) ; \quad 0 \leq t \leq T \\
p(T) & =\frac{\partial g}{\partial x}(X(T), Y(T))+E\left[\frac{\partial g}{\partial y}(X(T), Y(T))\right] \nabla_{X(T)} F .
\end{aligned}\right.
$$


Note that since we identify $\nabla_{X(t)}$ and $\nabla_{X(T)}$ as elements in $L^{2}(\Omega, P)$, the above equation is well-defined as a finite dimensional backward stochastic differential equation. See also equation (4.6) below and other places.

Existence and uniqueness results for this type (3.1) \& (3.7) of forward-backward SDEs with singular drift seem to an unexplored area of research. However, some partial results can be found in the recent paper [7].

Theorem 3.2 (Sufficient maximum principle for mean-field singular control.). Let $\hat{\xi}, \hat{u} \in \mathcal{A}$ be such that the system of (3.1) and (3.7) has a solution $\hat{X}(t), \hat{p}(t), \hat{q}(t)$ and set $\hat{Y}(t)=$ $F(\hat{X}(t, \cdot))$. Suppose the following conditions hold

- (The concavity assumptions)

The functions

$$
\begin{aligned}
& \mathbb{R}^{d} \times L^{2}(P) \times \mathbb{R}^{m} \times \mathbb{R}^{n} \ni(x, X, \xi, u) \rightarrow H(t, x, F(X), \xi, u, \hat{p}(t), \hat{q}(t), \omega) \\
& \text { and } \\
& X \rightarrow g(X, F(X)) \\
& \text { are concave for all } t \in[0, T] \text { and almost all } \omega \in \Omega \text {. }
\end{aligned}
$$

- (The conditional maximum property)

$$
\begin{aligned}
& \quad \underset{v \in A}{\operatorname{ssup} \sup } \mathbb{E}\left[H(t, \hat{X}(t), \hat{Y}(t), \hat{\xi}(t), v, \hat{p}(t), \hat{q}(t)) \mid \mathcal{G}_{t}\right] \\
= & \mathbb{E}\left[H(t, \hat{X}(t), \hat{Y}(t), \hat{\xi}(t), \hat{u}(t), \hat{p}(t), \hat{q}(t)) \mid \mathcal{G}_{t}\right] \text { a.s. }
\end{aligned}
$$

- (Variational inequality)

$$
\begin{aligned}
& \underset{\xi}{\operatorname{ess} \sup } \mathbb{E}\left[H(t, \hat{X}(t), \hat{Y}(t), \xi, \hat{u}(t), \hat{p}(t), \hat{q}(t)) \mid \mathcal{G}_{t}\right] \\
= & \mathbb{E}\left[H(t, \hat{X}(t), \hat{Y}(t), \hat{\xi}(t), \hat{u}(t), \hat{p}(t), \hat{q}(t)) \mid \mathcal{G}_{t}\right] \text { a.s. }
\end{aligned}
$$

Then $(\hat{\xi}(t), \hat{u}(t))$ is an optimal control for $J(\xi, u)$.

Proof. This theorem is a straightforward consequence of Theorem 4.1 below. We refer to the proof there.

\subsection{A necessary maximum principle}

In the previous section we gave a verification theorem, stating that if a given control $(\hat{\xi}, \hat{u})$ satisfies (3.8)-(3.10), then it is indeed optimal for the singular mean field control problem. We now establish a partial converse, implying that if a control $(\hat{\xi}, \hat{u})$ is optimal for the singular 
mean field control problem, then it is a conditional critical point for the Hamiltonian.

To achieve this, we start with the setup of [16] as follows. For $(u, \xi) \in \mathcal{A}$, let $\mathcal{V}(\xi)$ denote the set of $\mathbb{G}$-adapted processes $\eta$ of finite variation such that there exists $\delta=\delta(\xi)>0$ satisfying

$$
(u, \xi+a \eta) \in \mathcal{A} \text { for all } a \in[0, \delta] .
$$

Then for $(u, \xi) \in \mathcal{A}$ and $\eta \in \mathcal{V}(\xi)$ we have, by our smoothness assumptions on the coefficients,

$$
\begin{aligned}
\lim _{a \rightarrow 0^{+}} & \frac{1}{a}(J(\xi+a \eta)-J(\xi))=\mathbb{E}\left[\int _ { 0 } ^ { T } \left\{\frac{\partial f}{\partial x}(t, X(t), Y(t), \xi(t), u(t)) Z(t)\right.\right. \\
& \left.+\frac{\partial f}{\partial y}(t, X(t), Y(t), \xi(t), u(t))\left\langle\nabla_{X(t)} F, Z(t)\right\rangle\right\} d t \\
& \left.+\int_{0}^{T} \frac{\partial f}{\partial \xi}(t, X(t), Y(t), \xi(t), u(t)) d \eta(t)\right] \\
& +\mathbb{E}\left[\frac{\partial g}{\partial x}(X(T), Y(T)) Z(T)+\frac{\partial g}{\partial y}(X(T), Y(T))\left\langle\nabla_{X(T)} F, Z(T)\right\rangle\right] \\
& +\mathbb{E}\left[\int_{0}^{T} \frac{\partial h}{\partial x}(t, X(t), u(t)) Z(t) d \xi(t)+\int_{0}^{T} h(t, X(t), u(t)) d \eta(t)\right]
\end{aligned}
$$

where

$$
Z(t):=\lim _{a \rightarrow 0^{+}} \frac{1}{a}\left(X^{(\xi+a \eta)}(t)-X^{(\xi)}(t)\right) .
$$

Note that by the chain rule we have

$$
\lim _{a \rightarrow 0^{+}} \frac{1}{a}\left(Y^{(\xi+a \eta)}(t)-Y^{(\xi)}(t)\right)=\lim _{a \rightarrow 0^{+}} \frac{1}{a}\left(F\left(X^{(\xi+a \eta)}(t)\right)-F\left(X^{(\xi)}(t)\right)\right)=\left\langle\nabla_{X(t)} F, Z(t)\right\rangle .
$$

Moreover,

$$
\begin{aligned}
d Z(t) & =\left(\frac{\partial b}{\partial x}(t) Z(t)+\frac{\partial b}{\partial y}(t)\left\langle\nabla_{X(t)} F, Z(t)\right\rangle+\frac{\partial b}{\partial \xi}(t) \eta(t)\right) d t \\
& +\left(\frac{\partial \sigma}{\partial x}(t) Z(t)+\frac{\partial \sigma}{\partial y}(t)\left\langle\nabla_{X(t)} F, Z(t)\right\rangle+\frac{\partial \sigma}{\partial \xi}(t) \eta(t)\right) d B(t) \\
& +\frac{\partial \lambda}{\partial x}(t) Z(t) d \xi(t)+\lambda(t) d \eta(t) ; Z(0)=0
\end{aligned}
$$

where we did not write the explicit dependence of the functions $b, \sigma$ and $\lambda$ on their arguments $X, Y, \xi$, and $\eta$ for simplicity.

Remark 3.3. It is not obvious that the limit in (3.13) exists. We refer to [24] for a study of this issue in a related setting. Assuming that the limit exists, the computation leading to (3.15) is straightforward. For details see e.g. page 469 in [19].

We can now state and prove a necessary maximum principle: 
Theorem 3.4 (Necessary maximum principle for mean-field singular control). Suppose $(\hat{\xi}, \hat{u}) \in \mathcal{A}$ is optimal, i.e. satisfies (3.4). Suppose the following processes $\eta_{i}(s), i=$ $1,2,3$ belong to $\mathcal{V}(\hat{\xi})$ :

$$
\begin{aligned}
& \eta_{1}(s):=\alpha(\omega) \chi_{[t, T]}(s), \text { where } \alpha>0 \text { is } \mathcal{G}_{t}-\text { measurable, for all } t \in[0, T], \\
& \eta_{2}(s):=\hat{\xi}(s), \\
& \eta_{3}(s):=-\hat{\xi}(s), s \in[0, T] .
\end{aligned}
$$

Then

$$
\mathbb{E}\left[\frac{\partial H}{\partial u}(t, \hat{X}(t), \hat{Y}(t), \hat{\xi}(t), u, \hat{p}(t), \hat{q}(t))_{u=\hat{u}(t)} \mid \mathcal{G}_{t}\right]=0 \text { a.s. }
$$

Moreover, the following variational inequalities hold.

$$
\left\{\begin{array}{l}
\mathbb{E}\left[\frac{\partial H_{0}}{\partial \xi}(t, \hat{X}(t), \hat{Y}(t), \hat{\xi}(t), \hat{u}(t))+\lambda(t, \hat{X}(t), \hat{u}(t)) \hat{p}(t)+h(t, \hat{X}(t), \hat{u}(t)) \mid \mathcal{G}_{t}\right] \leq 0 \\
\text { for all } t \in[0, T] \text { a.s. } \quad \text { and } \\
\mathbb{E}\left[\frac{\partial H_{0}}{\partial \xi}(t, \hat{X}(t), \hat{Y}(t), \hat{\xi}(t), \hat{u}(t))+\lambda(t, \hat{X}(t), \hat{u}(t)) \hat{p}(t)+h(t, \hat{X}(t), \hat{u}(t)) \mid \mathcal{G}_{t}\right] d \hat{\xi}(t)=0 \\
\text { for all } t \in[0, T] \text { a.s. }
\end{array}\right.
$$

Proof. The proof of (3.16) is analogous to the non singular mean field control case and is omitted here. We shall prove (3.17) in the case when there is no control $u$. We need to prove that if $\hat{\xi} \in \mathcal{A}$ is optimal, i.e. if

$$
\sup _{\xi \in \mathcal{A}} J(\xi)=J(\hat{\xi})
$$

then $\hat{\xi}$ satisfies (3.17). To this end, choose $\xi \in \mathcal{A}$ and $\eta \in \mathcal{V}(\xi)$ and compute

$$
\left.\frac{d}{d a} J(\xi+a \eta)\right|_{a=0}=A_{1}+A_{2}+A_{3}+A_{4},
$$

where

$$
\begin{aligned}
& A_{1}=\mathbb{E}\left[\int_{0}^{T}\left\{\frac{\partial f}{\partial x}(t) Z(t)+\frac{\partial f}{\partial y}(t)\left\langle\nabla_{X(t)} F, Z(t)\right\rangle\right\} d t\right] \\
& A_{2}=\mathbb{E}\left[\int_{0}^{T} \frac{\partial f}{\partial \xi}(t) d \eta(t)\right] \\
& A_{3}=\mathbb{E}\left[\frac{\partial g}{\partial x}(X(T), Y(T)) Z(T)+\frac{\partial g}{\partial y}(X(T), Y(T))\left\langle\nabla_{X(T)} F, Z(T)\right\rangle\right] \\
& A_{4}=\mathbb{E}\left[\int_{0}^{T} \frac{\partial h}{\partial x}(t) Z(t) \xi(d t)+h(t) d \eta(t)\right] .
\end{aligned}
$$


By the definition of $H_{0}$ we have

$$
\begin{aligned}
A_{1} & =\mathbb{E}\left[\int_{0}^{T} Z(t)\left\{\frac{\partial H_{0}}{\partial x}(t)-\frac{\partial b}{\partial x}(t) p(t)-\frac{\partial \sigma}{\partial x}(t) q(t)\right\} d t\right. \\
& \left.+\int_{0}^{T}\left\langle\nabla_{X(t)} F, Z(t)\right\rangle\left(\frac{\partial H_{0}}{\partial y}(t)-\frac{\partial b}{\partial y}(t) p(t)-\frac{\partial \sigma}{\partial y}(t) q(t)\right) d t\right] .
\end{aligned}
$$

By the terminal condition of $p(T)$ (see (3.7)) and then by the Itô formula we have

$$
\begin{aligned}
A_{3}= & \mathbb{E}[p(T) Z(T)] \\
= & \mathbb{E}\left[\int_{0}^{T} p(t) d Z(t)+\int_{0}^{T} Z(t) d p(t)+\int_{0}^{T} q(t)\left(\frac{\partial \sigma}{\partial x}(t) Z(t)+\frac{\partial \sigma}{\partial y}(t)\left\langle\nabla_{X(t)} F, Z(t)\right\rangle+\frac{\partial \sigma}{\partial \xi}(t) \eta(t)\right) d t\right. \\
= & \mathbb{E}\left[\int_{0}^{T} p(t)\left\{\frac{\partial b}{\partial x}(t) Z(t)+\frac{\partial b}{\partial y}(t)\left\langle\nabla_{X(t)} F, Z(t)\right\rangle+\frac{\partial b}{\partial \xi}(t) \eta(t)\right\} d t\right. \\
& +\int_{0}^{T} p(t) \frac{\partial \lambda}{\partial x}(t) Z(t) d \xi(t)+\int_{0}^{T} p(t) \lambda(t) d \eta(t)-\int_{0}^{T}\left(\frac{\partial H_{0}}{\partial x}(t) Z(t)+\frac{\partial H_{0}}{\partial y}(t)\left\langle\nabla_{X(t)} F, Z(t)\right\rangle\right) d t \\
- & \int_{0}^{T} Z(t)\left\{\frac{\partial \lambda}{\partial x}(t) p(t)+\frac{\partial h}{\partial x}(t)\right\} d \xi(t)+\int_{0}^{T} q(t)\left(\frac{\partial \sigma}{\partial x}(t) Z(t)+\frac{\partial \sigma}{\partial y}(t)\left\langle\nabla_{X(t)} F, Z(t)\right\rangle\right) d t .
\end{aligned}
$$

Combining (3.19)-(3.22) we get

$$
\left.\frac{d}{d a} J(\xi+a \eta)\right|_{a=0}=\mathbb{E}\left[\int_{0}^{T}\left\{\frac{\partial H_{0}}{\partial \xi}(s)+\lambda(s) p(s)+h(s)\right\} d \eta(s)\right] .
$$

In particular, if we apply this to an optimal $\xi=\hat{\xi}$ for $J$ we get, for all $\eta \in \mathcal{V}(\hat{\xi})$,

$$
\mathbb{E}\left[\int_{0}^{T}\left\{\frac{\partial \hat{H}_{0}}{\partial \xi}(s)+\hat{\lambda}(t) \hat{p}(s)+\hat{h}(s)\right\} d \eta(s)\right]=\frac{d}{d a} J(\hat{\xi}+a \eta)_{a=0} \leq 0 .
$$

where we have denoted $\hat{H}_{0}(t):=H_{0}(t, \hat{X}(t), \hat{Y}(t), \hat{\xi}(t), \hat{u}(t), \hat{p}(t), \hat{q}(t)) ; \quad \hat{\lambda}(t):=\lambda(t, \hat{X}(t), \hat{u}(t))$; and $\hat{h}(t):=h(t, \hat{X}(t), \hat{u}(t))$.

If we choose $\eta$ to be a pure jump process of the form

$$
\eta(s)=\sum_{0<t_{i} \leq s} \alpha\left(t_{i}\right)
$$

where $\alpha(s)>0$ is $\mathcal{G}_{s}$-measurable for all $s,(3.23)$ gives

$$
\mathbb{E}\left[\left(\frac{\partial \hat{H}_{0}}{\partial \xi}(t)+\hat{\lambda}(t) \hat{p}(t)+\hat{h}(t)\right) \alpha\left(t_{i}\right)\right] \leq 0 \text { for all } t_{i} \text { a.s. }
$$


Since this holds for all such $\eta$ with arbitrary $t_{i}$, we conclude that

$$
\mathbb{E}\left[\frac{\partial \hat{H}_{0}}{\partial \xi}(t)+\hat{\lambda}(t) \hat{p}(t)+\hat{h}(t) \mid \mathcal{G}_{t}\right] \leq 0 \text { for all } t \in[0, T] \text { a.s. }
$$

Finally, applying (3.23) to

$$
d \eta(t)=d \hat{\xi}(t) \in \mathcal{V}(\hat{\xi})
$$

and then to

$$
d \eta(t)=-d \hat{\xi}(t) \in \mathcal{V}(\hat{\xi})
$$

we get, for all $t \in[0, T]$,

$$
\mathbb{E}\left[\frac{\partial \hat{H}_{0}}{\partial \xi}(t)+\hat{\lambda}(t) \hat{p}(t)+\hat{h}(t) \mid \mathcal{G}_{t}\right] d \hat{\xi}(t)=0 \text { for all } t \in[0, T] \text { a.s. } .
$$

With (3.24) and (3.25) the proof is complete.

\subsection{The optimality conditions}

Since there have already been studies (see e.g. [15] and references therein) on the usual (nonsingular) mean field control problems, let us consider only the singular control $\xi$, i.e. without regular control $u$. More precisely, the system that we deal with, is described by

$$
d X(t)=b(t, X(t), Y(t), \xi(t)) d t+\sigma(t, X(t), Y(t), \xi(t)) d B(t)+\lambda(t, X(t)) d \xi(t),
$$

with $Y(t)=F(X(t, \cdot))$. The performance functional is

$$
J(\xi)=\mathbb{E}\left[\int_{0}^{T} f(t, X(t), Y(t), \xi(t)) d t+g(X(T), Y(T))+\int_{0}^{T} h(t, X(t)) d \xi(t)\right] .
$$

We also assume that the full information is available to the controller. If we apply the Theorem 3.2 to the above singular control problem, we have the following description for the optimal control.

The auxiliary backward stochastic differential equation with mean field is

$$
\left\{\begin{aligned}
d p(t) & =-\left[\frac{\partial f}{\partial x}(t, X(t), Y(t), \xi(t))+\frac{\partial b}{\partial x}(t, X(t), Y(t), \xi(t)) p(t)\right. \\
& \left.+\frac{\partial \sigma}{\partial x}(t, X(t), Y(t), \xi(t)) q(t)\right] d t-\mathbb{E}\left[\frac{\partial f}{\partial y}(t, X(t), Y(t), \xi(t))\right. \\
& \left.+\frac{\partial b}{\partial y}(t, X(t), Y(t), \xi(t)) p(t)+\frac{\partial \sigma}{\partial y}(t, X(t), Y(t), \xi(t)) q(t)\right] \nabla_{X(t)} F d t \\
& -\left\{\frac{\partial \lambda}{\partial x}(t, X(t)) p(t)+\frac{\partial h}{\partial x}(t, X(t))\right\} \xi(d t)+q(t) d B(t), \\
p(T) & =\frac{\partial g}{\partial x}(X(T), Y(T))+\mathbb{E}\left[\frac{\partial g}{\partial y}(X(T), Y(T))\right] \nabla_{X(T)} F .
\end{aligned}\right.
$$


We denote

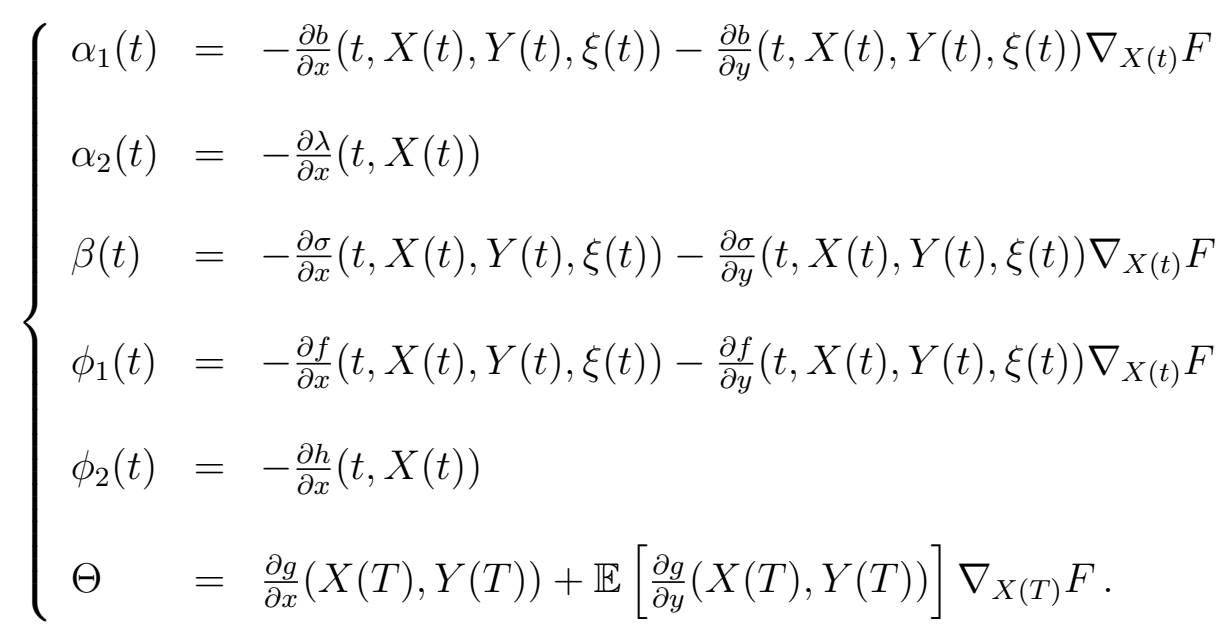

We also denote, for $0 \leq t \leq r \leq T$

$$
\rho_{t, r}:=\exp \left\{\int_{t}^{r} \beta(s) d W_{s}+\int_{t}^{r}\left[\alpha_{1}(s)-\frac{1}{2} \beta^{2}(s)\right] d s+\int_{t}^{r} \alpha_{2}(s) d \xi(s)\right\} .
$$

Then a straightforward application of Equation (2.11) of [11] to the above BSDE yields

$$
p(t)=\mathbb{E}\left(\Theta \rho_{t, T}+\int_{t}^{T} \rho_{t, r} \phi_{1}(r) d r+\int_{t}^{T} \rho_{t, r} \phi_{2}(r) d \xi(r) \mid \mathcal{F}_{t}\right) .
$$

With this $p$ and $q$, the optimal control for the above problem satisfies the following equations

$$
\left\{\begin{array}{l}
\frac{\partial f}{\partial \xi}(t, X(t), Y(t), \xi(t))+\frac{\partial b}{\partial \xi}(t, X(t), Y(t), \xi(t)) p(t)+\frac{\partial \sigma}{\partial \xi}(t, X(t), Y(t), \xi(t)) q(t) \\
\quad+\lambda(t, X(t)) p(t)+h(t, X(t)) \leq 0 \text { for all } t \in[0, T], \text { a.s. and } \\
\quad\left[\begin{array}{l}
\frac{\partial f}{\partial \xi}(t, X(t), Y(t), \xi(t))+\frac{\partial b}{\partial \xi}(t, X(t), Y(t), \xi(t)) p(t)+\frac{\partial \sigma}{\partial \xi}(t, X(t), Y(t), \xi(t)) q(t) \\
\quad+\lambda(t, X(t)) p(t)+h(t, X(t))] d \xi(t)=0 \text { for all } t \in[0, T] \text { a.s. }
\end{array}\right.
\end{array}\right.
$$

We obtain an equation which describes the domain $D$ in which the process $X(t)$ must live all the time. We control the process $X(t)$ in such a way that when the process is in the interior of the domain $D$, we don't do anything. When the process reaches the boundary of $D$, we exercise the minimal push to keep the process inside the domain $D$.

Equation (3.31) are essentially an equation for the "domain" of the state $X(t)$ and the condition for the singular control $\xi$ to satisfy. It can be complicated since the solution $p(t)$ may depend on the paths of $X, Y$ and the path of control $\xi$ itself up to time $t$. Denote

$$
X_{t}:=(X(s), 0 \leq s \leq t)
$$

the trajectory of $X$ up to time $t$, and similarly for $Y_{t}$ and $\xi_{t}$. Then $p(t)$ can be represented in general as $p(t)=p\left(X_{t}, Y_{t}, \xi_{t}\right)$. 
We now consider a slightly more general situation, where the singular control may be any finite variation process, not necessarily increasing. The increasing case corresponds to $r\left(t, X_{t}, Y_{t}, \xi_{t}\right)=\infty$ below.

Suppose that there are two functionals $l, r:[0, T] \times C([0, T], \mathbb{R})^{3} \rightarrow \mathbb{R}$ with $l \leq r$ such that the equations (3.31) can be written as

$$
\left\{\begin{array}{l}
l\left(t, X_{t}, Y_{t}, \xi_{t}\right) \leq X(t) \leq r\left(t, X_{t}, Y_{t}, \xi_{t}\right) \\
\int_{0}^{T}\left[X(t)-l\left(t, X_{t}, Y_{t}, \xi_{t}\right)\right] d \xi(t)=0 \\
\int_{0}^{T}\left[r\left(t, X_{t}, Y_{t}, \xi_{t}\right)-X(t)\right] d \xi(t)=0 .
\end{array}\right.
$$

Then we are led to the problem of finding a finite variation (not necessarily increasing) control $\xi$ for the system

$$
d X(t)=b(t, X(t), Y(t), \xi(t)) d t+\sigma(t, X(t), Y(t), \xi(t)) d B(t)+\lambda(t, X(t)) d \xi(t)
$$

satisfying (3.32). This is a Skorohod type problem.

For simplicity, we restrict ourselves to the case when

$$
\lambda(t, x)=1
$$

Theorem 3.5. Suppose that the following hold

1. $b$ and $\sigma$ are uniformly Lipschitz continuous, that is there exists $L>0$ such that

$$
\left|b\left(t, x_{2}, y_{2}, \xi_{2}\right)-b\left(t, x_{1}, y_{1}, \xi_{1}\right)\right| \leq L\left(\left|x_{2}-x_{1}\right|+\left|y_{2}-y_{1}\right|+\left|\xi_{2}-\xi_{1}\right|\right) \text {. }
$$

The same inequality holds for $\sigma$.

2. $l$ and $r$ are uniformly Lipschitz continuous, i.e. for some $L>0$,

$$
\begin{aligned}
& \left|r\left(t, X_{t}^{2}, Y_{t}^{2}, \xi_{t}^{2}\right)-r\left(t, X_{t}^{1}, Y_{t}^{1}, \xi_{t}^{1}\right)\right| \leq \kappa \sup _{0 \leq s \leq t}\left[\left|X^{2}(s)-X^{1}(s)\right|+\left|\xi^{2}(s)-\xi^{1}(s)\right|\right] \\
& \quad+L \int_{0}^{t} \sup _{0 \leq s \leq r}\left[\left|X^{2}(s)-X^{1}(s)+\right| Y^{2}(s)-Y^{1}(s)|+| \xi^{2}(s)-\xi^{1}(s) \mid\right] d r
\end{aligned}
$$

for some $0<\kappa<1 / 4$. The same inequality holds for $l$.

3. For any $t \in[0, T], X, Y$ and $\xi$ in $C([0, T], \mathbb{R})$,

$$
l\left(t, X_{t}, Y_{t}, \xi_{t}\right)<r\left(t, X_{t}, Y_{t}, \xi_{t}\right) .
$$

Then, Equations (3.32)-(3.33) have a unique solution. 
Proof. We shall apply the Banach fixed point theorem. Let $\mathbb{B}$ denote the Banach space of all continuous adapted processes $(X(t), \xi(t))$ which are square integrable. More precisely,

$$
\begin{aligned}
\mathbb{B}=\{ & (X, \xi), X \text { and } \xi \text { are continuous and adapted and } \\
& \left.\|(X, \xi)\|_{\mathbb{B}}:=\left\{\mathbb{E} \sup _{0 \leq t \leq T}\left(|X(t)|^{2}+|\xi(t)|^{2}\right)\right\}^{1 / 2}<\infty\right\} .
\end{aligned}
$$

From (3.32) and (3.33), we define the following mapping on $\mathbb{B}: F(X, \xi)=(Z, \eta)$, where $(Z, \eta)$ satisfies the inequalities

$$
\left\{\begin{array}{l}
l\left(t, X_{t}, Y_{t}, \xi_{t}\right) \leq Z(t) \leq r\left(t, X_{t}, Y_{t}, \xi_{t}\right) \\
\int_{0}^{T}\left[X(t)-l\left(t, X_{t}, Y_{t}, \xi_{t}\right)\right] d \eta(t)=0 \\
\int_{0}^{T}\left[r\left(t, X_{t}, Y_{t}, \xi_{t}\right)-X(t)\right] d \eta(t)=0,
\end{array}\right.
$$

and, in addition,

$$
d Z(t)=b(t, X(t), Y(t), \xi(t)) d t+\sigma(t, X(t), Y(t), \xi(t)) d B(t)+d \eta(t) .
$$

For a given continuous pair $(X(t), \xi(t))$ in $\mathbb{B}$, the problem to find a pair $(Z(t), \eta(t))$ such that (3.37), (3.38) hold is an example of a Skorohod reflection problem. See e.g. [8]. By condition (3), Theorem 2.6 and Corollary 2.4 of [4] the above Skorohod problem has a unique solution $(Z(t), \eta(t))$ and the solution pair $(Z(t), \eta(t))$ can be represented as

$$
\begin{aligned}
& \eta(t)=\Xi(l, r, \psi)(t) \\
& Z(t)=\psi(t)-\eta(t),
\end{aligned}
$$

where

$$
\begin{aligned}
& \psi(t):= \int_{0}^{t} b(s, X(s), Y(s), \xi(s)) d s+\int_{0}^{t} \sigma(s, X(s), Y(s), \xi(s)) d B(s) \\
& l(u):=l\left(u, X_{u}, Y_{u}, \xi_{u}\right) \\
& r(u):=r\left(u, X_{u}, Y_{u}, \xi_{u}\right) \\
& \Xi(l, r, \psi)(t):=\max \left\{\left[(\psi(0)-r(0))^{+} \wedge \inf _{u \in[0, t]}(\psi(u)-l(u))\right],\right. \\
&\left.\sup _{s \in[0, t]}\left[(\psi(s)-r(s)) \wedge \inf _{u \in[s, t]}(\psi(u)-l(u))\right]\right\} .
\end{aligned}
$$

It is elementary to see that

$$
\begin{aligned}
& \left|\max \left\{b_{1}, b_{2}\right\}-\max \left\{a_{1}, a_{2}\right\}\right| \leq \max \left\{\left|b_{1}-a_{1}\right|,\left|b_{2}-a_{2}\right|\right\} \\
& \left|\sup _{0 \leq t \leq T} g(t)-\sup _{0 \leq t \leq T} f(t)\right| \leq \sup _{0 \leq t \leq T}|g(t)-f(t)| \\
& \left|\inf _{0 \leq t \leq T} g(t)-\inf _{0 \leq t \leq T} f(t)\right| \leq \sup _{0 \leq t \leq T}|g(t)-f(t)| .
\end{aligned}
$$


From the expression of $\Xi$, we get that

$$
\begin{gathered}
\sup _{0 \leq s \leq t}\left|\Xi\left(l_{2}, r_{2}, \psi_{2}\right)(s)-\Xi\left(l_{1}, r_{1}, \psi_{1}\right)(s)\right| \leq 2 \sup _{0 \leq s \leq t}\left[\left|l_{2}(s)-l_{1}(s)\right|+\left|r_{2}(s)-r_{1}(s)\right|\right] \\
+4 \sup _{0 \leq s \leq t}\left[\left|\psi_{2}(s)-\psi_{1}(s)\right|\right] .
\end{gathered}
$$

Now we want to show that

$$
\mathbb{B} \ni(X, \xi) \rightarrow F(X, \xi)=(Z, \eta)
$$

is a contraction on $\mathbb{B}$. Assume that $\left(X^{1}, \xi^{1}\right)$ and $\left(X^{2}, \xi^{2}\right)$ be two elements in $\mathbb{B}$ and let $\left(Z^{1}, \eta^{1}\right)$ and $\left(Z^{2}, \eta^{2}\right)$ be the corresponding solutions to (3.37)-(3.38). Then for $i=1,2$, we have

$$
\begin{aligned}
& \eta_{i}(t)=\Xi\left(l_{i}, r_{i}, \psi_{i}\right)(t) \\
& Z_{i}(t)=\psi_{i}(t)-\eta_{i}(t),
\end{aligned}
$$

where

$$
\begin{aligned}
& \psi_{i}(t):=\int_{0}^{t} b\left(s, X^{i}(s), Y^{i}(s), \xi^{i}(s)\right) d s+\int_{0}^{t} \sigma_{i}\left(s, X^{i}(s), Y^{i}(s), \xi^{i}(s)\right) d B(s) \\
& l_{i}(u):=l\left(u, X_{u}^{i}, Y_{u}^{i}, \xi_{u}^{i}\right) \\
& r_{i}(u):=r\left(u, X_{u}^{i}, Y_{u}^{i}, \xi_{u}^{i}\right) \\
& \eta_{i}:=\Xi\left(l_{i}, r_{i}, \psi_{i}\right)(t)=\max \left\{\left[\left(\psi_{i}(0)-r_{i}(0)\right)^{+} \wedge \inf _{u \in[0, t]}\left(\psi_{i}(u)-l_{i}(u)\right)\right],\right. \\
&\left.\sup _{s \in[0, t]}\left[\left(\psi_{i}(s)-r_{i}(s)\right) \wedge \inf _{u \in[s, t]}\left(\psi_{i}(u)-l_{i}(u)\right)\right]\right\} .
\end{aligned}
$$

From (3.41) and then from the assumptions on $l$ and $r$, we see that

$$
\begin{aligned}
& \mathbb{E} \sup _{0 \leq r \leq t}\left|\eta_{2}(r)-\eta_{1}(r)\right|^{2} \\
\leq & 8 \mathbb{E} \sup _{0 \leq s \leq t}\left[\left|l_{2}(s)-l_{1}(s)\right|^{2}+\left|r_{2}(s)-r_{1}(s)\right|^{2}\right]+32 \mathbb{E} \sup _{0 \leq s \leq t}\left[\left|\psi_{2}(s)-\psi_{1}(s)\right|^{2}\right] \\
\leq & 8 \kappa^{2} \mathbb{E} \sup _{0 \leq r \leq t}\left[\left|X^{2}(r)-X^{1}(r)\right|^{2}+\left|\xi^{2}(r)-\xi^{1}(r)\right|^{2}\right]+32 \mathbb{E} \sup _{0 \leq s \leq t}\left|\psi_{2}(s)-\psi_{1}(s)\right|^{2} \\
& \quad+C \int_{0}^{t} \mathbb{E} \sup _{0 \leq r \leq t}\left[\left|X^{2}(r)-X^{1}(r)\right|^{2}+\left|\xi^{2}(r)-\xi^{1}(r)\right|^{2}\right] d r .
\end{aligned}
$$

By standard argument from stochastic analysis, we have

$$
\mathbb{E} \sup _{0 \leq s \leq t}\left|\psi_{2}(s)-\psi_{1}(s)\right|^{2} \leq C \int_{0}^{t} \mathbb{E} \sup _{0 \leq s \leq r}\left[\left|X^{2}(s)-X^{1}(s)\right|^{2}+\left|\xi^{2}(s)-\xi^{1}(s)\right|^{2}\right] d r .
$$


Thus we have

$$
\begin{aligned}
\mathbb{E} \sup _{0 \leq r \leq t}\left|\eta_{2}(r)-\eta_{1}(r)\right|^{2} \leq & 8 \mathbb{E} \sup _{0 \leq s \leq t}\left[\left|l_{2}(s)-l_{1}(s)\right|^{2}+\left|r_{2}(s)-r_{1}(s)\right|^{2}\right] \\
& +C \int_{0}^{t} \mathbb{E} \sup _{0 \leq s \leq r}\left[\left|X^{2}(s)-X^{1}(s)\right|^{2}+\left|\xi^{2}(s)-\xi^{1}(s)\right|^{2}\right] d r .
\end{aligned}
$$

From (3.43) we have

$$
\begin{aligned}
\mathbb{E} \sup _{0 \leq r \leq t}\left|Z_{2}(r)-Z_{1}(r)\right|^{2} \leq & 2 \mathbb{E} \sup _{0 \leq r \leq t}\left|\eta_{2}(r)-\eta_{1}(r)\right|^{2}+2 \mathbb{E} \sup _{0 \leq r \leq t}\left|\psi_{2}(r)-\psi_{1}(r)\right|^{2} \\
\leq & 16 \kappa^{2} \mathbb{E} \sup _{0 \leq r \leq t}\left[\left|X^{2}(r)-X^{1}(r)\right|^{2}+\left|\xi^{2}(r)-\xi^{1}(r)\right|^{2}\right] \\
& +64 \mathbb{E} \sup _{0 \leq s \leq t}\left|\psi_{2}(s)-\psi_{1}(s)\right|^{2} \\
& +C \int_{0}^{t} \mathbb{E} \sup _{0 \leq s \leq r}\left[\left|X^{2}(s)-X^{1}(s)\right|^{2}+\left|\xi^{2}(s)-\xi^{1}(s)\right|^{2}\right] d r \\
\leq & 16 \kappa^{2} \mathbb{E} \sup _{0 \leq r \leq t}\left[\left|X^{2}(r)-X^{1}(r)\right|^{2}+\left|\xi^{2}(r)-\xi^{1}(r)\right|^{2}\right] \\
& +C \int_{0}^{t} \mathbb{E} \sup _{0 \leq s \leq r}\left[\left|X^{2}(s)-X^{1}(s)\right|^{2}+\left|\xi^{2}(s)-\xi^{1}(s)\right|^{2}\right] d r .
\end{aligned}
$$

Combining the above inequality with (3.44), we have

$$
\begin{aligned}
& \mathbb{E} \sup _{0 \leq r \leq t}\left[\left|Z_{2}(r)-Z_{1}(r)\right|^{2}+\left|\eta_{2}(r)-\eta_{1}(r)\right|^{2}\right] \\
& \leq \quad 16 \kappa^{2} \mathbb{E} \sup _{0 \leq r \leq t}\left[\left|X^{2}(r)-X^{1}(r)\right|^{2}+\left|\xi^{2}(r)-\xi^{1}(r)\right|^{2}\right] \\
&+C \int_{0}^{t} \mathbb{E} \sup _{0 \leq s \leq r}\left[\left|X^{2}(s)-X^{1}(s)\right|^{2}+\left|\xi^{2}(s)-\xi^{1}(s)\right|^{2}\right] d r \\
& \leq \quad\left(16 \kappa^{2}+C t\right) \mathbb{E} \sup _{0 \leq r \leq t}\left[\left|X^{2}(r)-X^{1}(r)\right|^{2}+\left|\xi^{2}(r)-\xi^{1}(r)\right|^{2}\right] .
\end{aligned}
$$

If $\kappa<1 / 4$, then we can choose $t_{0}$ such that $16 \kappa^{2}+C t<1$ for all $t \leq t_{0}$. Thus from (3.45), we conclude that $F$ is a contraction mapping from $\mathbb{B}$ to $\mathbb{B}$. Following a routine argument, we obtain the solution $\xi(t), X(t)$ of equations (3.32) and (3.33) up to time $t_{0}$. Since the constant $C$ in $16 \kappa^{2}+C t$ does not depend on the initial condition, we repeat this procedure to solve the equations (3.32) and (3.33) on the interval $[0, T]$.

Remark 3.6. From the proof of the theorem, we see that if we define the Picard iteration 
for $n=0,1,2, \cdots$,

$$
\left\{\begin{array}{l}
l\left(t, X_{t}^{(n)}, Y_{t}^{(n)}, \xi_{t}^{(n)}\right) \leq X^{(n+1)}(t) \leq r\left(t, X_{t}^{(n)}, Y_{t}^{(n)}, \xi_{t}^{(n)}\right) \\
\int_{0}^{T}\left[X^{(n)}(t)-l\left(t, X_{t}^{(n)}, Y_{t}^{(n)}, \xi_{t}^{(n)}\right)\right] d \xi^{(n+1)}(t)=0 \\
\int_{0}^{T}\left[r\left(t, X_{t}^{(n)}, Y_{t}^{(n)}, \xi_{t}^{(n)}\right)-X^{(n)}(t)\right] d \xi^{(n+1)}(t)=0
\end{array}\right.
$$

and

$$
\begin{aligned}
d X^{(n+1)}(t)= & b(t, \\
& \left.X^{(n)}(t), Y^{(n)}(t), \xi^{(n)}(t)\right) d t \\
& +\sigma\left(t, X^{(n)}(t), Y^{(n)}(t), \xi^{(n)}(t)\right) d B(t)+d \xi^{(n+1)}(t),
\end{aligned}
$$

where $X_{t}^{(0)}=X(0), \xi_{t}^{(0)}=0$, then $\left(X^{(n)}(t), \xi^{(n)}(t)\right)$ will converge to the solution $(X(t), \xi(t))$ in $\mathbb{B}$. This may be used to construct numerical solutions.

\subsection{Application to optimal harvesting problem}

Let us consider an optimal harvesting problem where the population density $X(t)$ at time $t$ is described by the linear controlled system

$$
d X(t)=\left(b_{1}(t) X(t)+b_{2}(t) \mathbb{E}[X(t)]\right) d t+\sigma(t, X(t), Y(t)) d B(t)-d \xi(t) .
$$

We want to find $\hat{\xi}$ such that

$$
\sup _{\xi \in \mathcal{A}} J(\xi)=J(\hat{\xi})
$$

where

$$
J(\xi)=\mathbb{E}\left[\int_{0}^{T} f(t, X(t), Y(t)) d t+g(X(T))+\int_{0}^{T} h(t, X(t)) d \xi(t)\right]
$$

with

$$
f(t, x, y):=f_{1}(t) x+f_{2}(t) y
$$

and

$$
g(x):=K x
$$

with $K>0$. Then from (3.29) we get

$$
\left\{\begin{array}{l}
\alpha(t)=-b_{1}(t)-b_{2}(t) \\
\beta(t)=-\frac{\partial \sigma}{\partial x}(t, X(t), Y(t))-\frac{\partial \sigma}{\partial y}(t, X(t), Y(t)) \\
\phi_{1}(t)=-f_{1}(t)-f_{2}(t) \\
\phi_{2}(t)=-\frac{\partial h}{\partial x}(t, X(t)) \\
\Theta \quad K
\end{array}\right.
$$


Denote, for $0 \leq t \leq r \leq T$,

$$
\rho_{t, r}=\exp \left\{\int_{t}^{r} \beta(s) d W_{s}+\int_{t}^{r}\left[\alpha(s) d s-\frac{1}{2} \beta^{2}(s)\right] d s\right\} .
$$

Since $\alpha$ is deterministic, we have for all $t \leq r \leq T$,

$$
\begin{aligned}
a(t, r) & :=\mathbb{E}\left(\rho_{t, r} \mid \mathcal{F}_{t}\right) \\
& =\exp \left\{\int_{t}^{r} \alpha(s) d s\right\} \mathbb{E}\left(\exp \left\{\int_{t}^{r} \beta(s) d W_{s}-\frac{1}{2} \int_{t}^{r} \beta^{2}(s) d s\right\} \mid \mathcal{F}_{t}\right) \\
& =\exp \left\{\int_{t}^{r} \alpha(s) d s\right\} .
\end{aligned}
$$

Note that $a(t, r)$ is a deterministic function. It is easy to see from (3.30) that

$$
p(t)=K a(t, T)+\int_{t}^{T} a(t, r) \phi_{1}(r) d r+\mathbb{E}\left[\int_{t}^{T} a(t, r) \phi_{2}(r) d \xi(r) \mid \mathcal{F}_{t}\right] .
$$

Thus we have the optimality conditions

$$
\left\{\begin{array}{l}
-p(t)+h(t, X(t)) \leq 0 \\
{[-p(t)+h(t, X(t))] d \xi(t)=0 .}
\end{array}\right.
$$

If furthermore we assume

$$
\phi(t) \geq 0
$$

and

$$
h(t, x)=h_{0}(t) x^{\kappa}, \text { with } h_{0}(t) \text { positive and } \kappa \neq 0
$$

we get

$$
X(t) \begin{cases}\leq\left(\frac{p(t)}{h_{0}(t)}\right)^{\frac{1}{\kappa}} & \text { if } \kappa>0 \\ \geq\left(\frac{h_{0}(t)}{p(t)}\right)^{-\frac{1}{\kappa}} & \text { if } \kappa<0\end{cases}
$$

In this case, we can take

$$
\left\{\begin{array}{lll}
l(t, x, y, \xi)=0 \quad \text { and } & r(t, x, y, \xi)=\left(\frac{p(t)}{h_{0}(t)}\right)^{\frac{1}{\kappa}} & \text { if } \kappa>0 \\
l(t, x, y, \xi)=\left(\frac{h_{0}(t)}{p(t)}\right)^{-\frac{1}{\kappa}} & \text { and } \quad r(t, x, y, \xi)=\infty & \text { if } \kappa<0 .
\end{array}\right.
$$

Note that $\kappa<0$ means that unit price goes up when the population goes down (which becomes more precious). In this case, we want to keep the population above a threshold $\bar{h}(t):=\left(\frac{h_{0}(t)}{p(t)}\right)^{-\frac{1}{\kappa}}$. It is interesting to note that when $h_{0}(t)$ is larger, this threshold $\bar{h}(t)$ is also larger. We have proved 
Proposition 3.7. Under the assumptions (3.50), (3.51), (3.54) and (3.55), the solution of the mean field singular control problem (3.49) is given by the solution $(\hat{X}, \hat{Y}, \hat{\xi})$ of the Skorohod reflection problem (3.32) and (3.33), with the boundaries $l$ and $r$ given by (3.56).

Next, we continue to assume (3.54). But we replace (3.55) by

$$
h(t, x, y)=h_{0}(t) x^{2}+h_{1}(t) x .
$$

where $h_{0}(t)$ is positive. Then, inequalities (3.53) become

$$
\begin{gathered}
-p(t)+h_{0}(t) X^{2}(t)+h_{1}(t) X(t) \leq 0 . \\
l(t) \leq X(t) \leq r(t),
\end{gathered}
$$

where

$$
\begin{aligned}
l(t) & :=\frac{h_{1}(t)-\sqrt{h_{1}^{2}(t)+4 h_{0}(t) p(t)}}{2 h_{0}(t)} \\
r(t) & :=\frac{h_{1}(t)+\sqrt{h_{1}^{2}(t)+4 h_{0}(t) p(t)}}{2 h_{0}(t)} .
\end{aligned}
$$

Similar to Proposition 3.7, we have

Proposition 3.8. Under the assumptions (3.50), (3.51), (3.54) and (3.57), the solution of the mean field singular control problem (3.49) is given by the solution $(\hat{X}, \hat{Y}, \hat{\xi})$ of the Skorohod reflection problem (3.32) and (3.33), with the boundaries $l$ and $r$ given by (3.58)(3.60).

If $h$ is given by (3.57), but with $h_{0}(t)<0$, then it can be shown that the domain (3.31) will be either $X(t) \leq \underline{\mathrm{h}}(t)$ or $X(t) \geq \bar{h}(t)$, where $\underline{\mathrm{h}}$ and $\bar{h}$ are some thresholds which can be computed similarly.

\section{Singular mean-field two-players games}

\subsection{Sufficient and necessary maximum principles}

In this section we consider the stochastic game of two players, each of them is to maximize his/her singular mean-field performance.

Denote $\xi=\left(\xi_{1}, \xi_{2}\right), u=\left(u_{1}, u_{2}\right), w=\left(w_{1}, w_{2}\right), \lambda=\left(\lambda_{1}, \lambda_{2}\right), h=\left(h_{1}, h_{2}\right)$ with $h_{i}=$ $\left(h_{i, 1}, h_{i, 2}\right)$, and let the pair $w_{i}=\left(\xi_{i}, u_{i}\right)$ represent the control of player $i ; i=1,2$.

Suppose the process $X(t)=X^{\xi, u}(t)$ under control of the two players satisfy the following stochastic differential equation with jumps.

$$
\begin{aligned}
d X(t) & =b(t, X(t), Y(t), \xi(t), u(t), \omega) d t+\sigma(t, X(t), Y(t), \xi(t), u(t), \omega) d B(t) \\
& +\lambda(t, X(t), u(t), \omega) d \xi(t),
\end{aligned}
$$


where $Y(t)=F(X(t, \cdot))$, and $F$ is a Fréchet differentiable operator on $L^{2}(P)$, and we assume the same dimensions as in the beginning of Section 3.1.

We put $\mathbb{G}^{i}=\left\{\mathcal{G}_{t}^{i}\right\}_{t \geq 0}$ where $\mathcal{G}_{t}^{i} \subseteq \mathcal{F}_{t}$ is the information available to player $i$ at time $t$. The performance functional for player $i$ is assumed to be on the form

$$
\begin{aligned}
J_{i}(\xi, u) & =\mathbb{E}\left[\int_{0}^{T} f_{i}(t, X(t), Y(t), w(t), \omega) d t+g_{i}(X(T), Y(T), \omega)\right. \\
& \left.+\int_{0}^{T} h_{i, 1}(t, X(t), u(t), \omega) d \xi_{1}(t)+\int_{0}^{T} h_{i, 2}(t, X(t), u(t), \omega) d \xi_{2}(t)\right] ; i=1,2 .
\end{aligned}
$$

We want to find a Nash equilibrium for this game, i.e. find $\left(\xi_{1}^{*}, u_{1}^{*}\right) \in \mathcal{A}_{1}$ and $\left(\xi_{2}^{*}, u_{2}^{*}\right) \in \mathcal{A}_{2}$ such that

$$
\sup _{\left(\xi_{1}, u_{1}\right) \in \mathcal{A}_{1}} J_{1}\left(\xi_{1}, u_{1}, \xi_{2}^{*}, u_{2}^{*}\right) \leq J_{1}\left(\xi_{1}^{*}, u_{1}^{*}, \xi_{2}^{*}, u_{2}^{*}\right)
$$

and

$$
\sup _{\left(\xi_{2}, u_{2}\right) \in \mathcal{A}_{2}} J_{2}\left(\xi_{1}^{*}, u_{1}^{*}, \xi_{2}, u_{2}\right) \leq J_{2}\left(\xi_{1}^{*}, u_{1}^{*}, \xi_{2}^{*}, u_{2}^{*}\right)
$$

Here $\mathcal{A}_{i}$ is a given family of $\mathbb{G}^{(i)}$-predictable processes such that the corresponding state equation has a unique solution $X$ such that $\omega \rightarrow X(t, \omega) \in L^{2}(P)$ for all t.

We let $A^{(i)}$ denote the set of possible values of $u_{i}(t) ; t \in[0, T]$ when $\left(\xi_{i}, u_{i}\right) \in \mathcal{A}_{i} ; i=1,2$.

We make similar assumptions as in Section 3.1, i.e. we assume that the functions $b, \sigma, \lambda_{j}, f_{i}, g_{i}$ and $h_{i, j}$ are progressively measurable with respect to $(t, \omega)$ for all fixed $x, y, \xi, u$ and continuously differentiable with respect to $x, y, \xi, u$ with bounded derivatives.

Define two Hamiltonians $H_{i} ; i=1,2$, as follows:

$$
\begin{aligned}
& H_{i}\left(t, x, y, \xi_{1}, u_{1}, \xi_{2}, u_{2}, p_{i}, q_{i}\right)\left(d t, d \xi_{1}(t), d \xi_{2}(t)\right) \\
& \quad=H_{i, 0}\left(t, x, y, \xi_{1}, u_{1}, \xi_{2}, u_{2}, p_{i}, q_{i}\right) d t+\sum_{j=1}^{2}\left\{\lambda_{j}(t, x, u) p_{i}+h_{i, j}(t, x, u)\right\} d \xi_{j}(t)
\end{aligned}
$$

where

$$
H_{i, 0}\left(t, x, y, w, p_{i}, q_{i}\right):=f_{i}(t, x, y, w)+b(t, x, y, \xi, u) p_{i}+\sigma(t, x, y, \xi, u) q_{i} .
$$

We assume that for $i=1,2, H=H_{i}$ is Fréchet differentiable $\left(C^{1}\right)$ in the variables $x, y, \xi, u$. 
The BSDE for the adjoint processes $p_{i}, q_{i}$ is

$$
\left\{\begin{aligned}
d p_{i}(t) & =-\frac{\partial H_{i, 0}}{\partial x}\left(t, X(t), Y(t), w(t), p_{i}(t), q_{i}(t)\right) d t \\
& -\mathbb{E}\left[\frac{\partial H_{i, 0}}{\partial y}\left(t, X(t), Y(t), w(t), p_{i}(t), q_{i}(t)\right)\right] \nabla_{X(t)} F d t \\
& -\sum_{j=1}^{2}\left\{\frac{\partial \lambda_{j}}{\partial x}(t, X(t), u(t)) p_{i}(t)+\frac{\partial h_{i, j}}{\partial x}(t, X(t), u(t))\right\} d \xi_{j}(t)+q_{i}(t) d B(t) \\
p_{i}(T)= & \frac{\partial g_{i}}{\partial x}(X(T), Y(T))+\mathbb{E}\left[\frac{\partial g_{i}}{\partial y}(X(T), Y(T))\right] \nabla_{X(T)} F ; \quad i=1,2 .
\end{aligned}\right.
$$

We now state a sufficient maximum principle for stochastic games with two players and mean field terms.

Theorem 4.1 (Sufficient maximum principle).

Let $\left(\hat{\xi}_{1}, \hat{u}_{1}\right) \in \mathcal{A}_{1},\left(\hat{\xi}_{2}, \hat{u}_{2}\right) \in \mathcal{A}_{2}$ with corresponding solutions $\hat{X}, \hat{p}_{i}, \hat{q}_{i}, \hat{r}_{i}$ of (4.1) and (4.6). Assume the following:

- The maps

$$
X, w_{1} \rightarrow H_{1}\left(t, X, F(X), w_{1}, \hat{w}_{2}(t), \hat{p}_{1}(t), \hat{q}_{1}(t)\right)
$$

and

$$
X, w_{2} \rightarrow H_{2}\left(t, X, F(X), \hat{w}_{1}, w_{2}(t), \hat{p}_{2}(t), \hat{q}_{2}(t)\right)
$$

and

$$
X \rightarrow g_{i}(X, F(X))
$$

are concave for all $t ; i=1,2$.

- (The conditional maximum properties)

$$
\begin{aligned}
\underset{u_{1} \in A_{1}}{\operatorname{ess} \sup } & \mathbb{E}\left[H_{1}\left(t, \hat{X}(t), \hat{Y}(t), \hat{\xi}_{1}(t), u_{1}, \hat{\xi}_{2}(t), \hat{u}_{2}(t), \hat{p}_{1}(t), \hat{q}_{1}(t)\right) \mid \mathcal{G}_{t}^{(1)}\right] \\
& =\mathbb{E}\left[H_{1}\left(t, \hat{X}(t), \hat{Y}(t), \hat{\xi}_{1}(t), \hat{u}_{1}(t), \hat{\xi}_{2}(t), \hat{u}_{2}(t), \hat{p}_{1}(t), \hat{q}_{1}(t)\right) \mid \mathcal{G}_{t}^{(1)}\right] \quad \text { a.s. }
\end{aligned}
$$

and

$$
\begin{aligned}
\underset{u_{2} \in A_{2}}{\operatorname{ess~sup}} & \mathbb{E}\left[H_{2}\left(t, \hat{X}(t), \hat{Y}(t), \hat{\xi}_{1}(t), \hat{u}_{1}(t), \hat{\xi}_{2}(t), u_{2}, \hat{p}_{2}(t), \hat{q}_{2}(t)\right) \mid \mathcal{G}_{t}^{(2)}\right] \\
& =\mathbb{E}\left[H_{2}\left(t, \hat{X}(t), \hat{Y}(t), \hat{\xi}_{1}(t), \hat{u}_{1}(t), \hat{\xi}_{2}(t), \hat{u}_{2}(t), \hat{p}_{2}(t), \hat{q}_{2}(t)\right) \mid \mathcal{G}_{t}^{(2)}\right], \quad \text { a.s. }
\end{aligned}
$$

- (Variational inequalities)

$$
\begin{aligned}
\underset{\xi_{1}}{e s s \sup } & \mathbb{E}\left[H_{1}\left(t, \hat{X}(t), \hat{Y}(t), \xi_{1}, \hat{u}_{1}(t), \hat{\xi}_{2}(t), \hat{u}_{2}(t), \hat{p}_{1}(t), \hat{q}_{1}(t)\right) \mid \mathcal{G}_{t}^{(1)}\right] \\
\quad= & \mathbb{E}\left[H_{1}\left(t, \hat{X}(t), \hat{Y}(t), \hat{\xi}_{1}(t), \hat{u}_{1}(t), \hat{\xi}_{2}(t), \hat{u}_{2}(t), \hat{p}_{1}(t), \hat{q}_{1}(t)\right) \mid \mathcal{G}_{t}^{(1)}\right] \quad \text { a.s. }
\end{aligned}
$$


and

$$
\begin{aligned}
\underset{\xi_{2}}{\operatorname{ess} \sup } & \mathbb{E}\left[H_{2}\left(t, \hat{X}(t), \hat{Y}(t), \hat{\xi}_{1}(t), \hat{u}_{1}(t), \xi_{2}, \hat{u}_{2}(t), \hat{p}_{2}(t), \hat{q}_{2}(t)\right) \mid \mathcal{G}_{t}^{(2)}\right] \\
& =\mathbb{E}\left[H_{2}\left(t, \hat{X}(t), \hat{Y}(t), \hat{\xi}_{1}(t), \hat{u}_{1}(t), \hat{\xi}_{2}(t), \hat{u}_{2}(t), \hat{p}_{2}(t), \hat{q}_{2}(t)\right) \mid \mathcal{G}_{t}^{(2)}\right], \quad \text { a.s. }
\end{aligned}
$$

Then $\left(\hat{\xi}_{1}, \hat{u}_{1}\right),\left(\hat{\xi}_{2}, \hat{u}_{2}\right)$ is a Nash equilibrium, in the sense that (4.2) and (4.3) hold with $\xi_{i}^{*}:=\hat{\xi}_{i}, u_{i}^{*}:=\hat{u}_{i} ; i=1,2$.

Proof. By introducing a suitable increasing sequence of stopping times converging to $T$, we see that we may assume that all local martingales appearing in the proof below are martingales. We refer to [18] for details. We first study the stochastic control problem (4.2). For simplicity of notation, in the following we put:

$$
X(t)=X^{\xi_{1}, u_{1}, \hat{\xi}_{2}, \hat{u}_{2}}(t), Y(t)=Y^{\xi_{1}, u_{1}, \hat{\xi}_{2}, \hat{u}_{2}}(t) \text { and } \hat{X}(t)=X^{\hat{\xi}_{1}, \hat{u}_{1}, \hat{\xi}_{2}, \hat{u}_{2}}(t), \hat{Y}(t)=Y^{\hat{\xi}_{1}, \hat{u}_{1}, \hat{\xi}_{2}, \hat{u}_{2}}(t)
$$
$b(t)=b\left(t, X(t), Y(t), \xi_{1}(t), \xi_{2}(t), u_{1}(t), u_{2}(t), \omega\right), \hat{b}(t)=b\left(t, \hat{X}(t), \hat{Y}(t), \xi_{1}(t), \hat{\xi}_{2}(t), u_{1}(t), \hat{u}_{2}(t), \omega\right)$ and similarly with $\sigma(t), \hat{\sigma}(t)$.

Consider $J_{1}\left(\xi_{1}, u_{1}, \hat{\xi}_{2}, \hat{u}_{2}\right)-J_{1}\left(\hat{\xi}_{1}, \hat{u}_{1}, \hat{\xi}_{2}, \hat{u}_{2}\right)=I_{1}+I_{2}+I_{3}+I_{4}$, where

$$
\begin{array}{r}
I_{1}:=\mathbb{E}\left[\int_{0}^{T}\left\{f_{1}\left(t, X(t), Y(t), w_{1}(t), \hat{w}_{2}(t)\right)-f_{1}\left(t, \hat{X}(t), \hat{Y}(t), \hat{w}_{1}(t), \hat{w}_{2}(t)\right)\right\} d t\right] \\
I_{2}:=\mathbb{E}\left[g_{1}(X(T), Y(T))-g_{1}(\hat{X}(T), \hat{Y}(T))\right] \\
I_{3}:=\mathbb{E}\left[\int_{0}^{T}\left\{h_{1,1}\left(t, X(t), w_{1}(t), \hat{w}_{2}(t)\right) d \xi_{1}(t)-h_{1,1}(t, \hat{X}(t), \hat{w}(t)) d \hat{\xi}_{1}(t)\right\}\right] \\
I_{4}:=\mathbb{E}\left[\int_{0}^{T}\left\{h_{1,2}\left(t, X(t), w_{1}(t), \hat{w}_{2}(t)\right) d \xi_{2}(t)-h_{1,2}(t, \hat{X}(t), \hat{w}(t)) d \hat{\xi}_{2}(t)\right\}\right]
\end{array}
$$

By the definition of $H_{1}$ we have

$$
\begin{aligned}
I_{1}=\mathbb{E}[ & \int_{0}^{T}\left\{H_{1,0}\left(t, X_{t}, Y_{t}, w_{1}(t), \hat{w}_{2}(t), \hat{p}_{1}(t), \hat{q}_{1}(t)\right)\right. \\
& \left.\left.\quad-H_{1,0}\left(t, \hat{X}_{t}, \hat{Y}_{t}, \hat{w}(t), \hat{p}_{1}(t), \hat{q}_{1}(t)\right)-(b(t)-\hat{b}(t)) \hat{p}_{1}(t)-(\sigma(t)-\hat{\sigma}(t)) \hat{q}_{1}(t)\right\} d t\right]
\end{aligned}
$$

By concavity of $g_{1}$ and the Itô formula we have

$$
\begin{aligned}
& I_{2} \leq \mathbb{E}[\left.\frac{\partial g_{1}}{\partial x}(\hat{X}(T), \hat{Y}(T))\right)(X(T)-\hat{X}(T)) \\
&\left.+\frac{\partial g_{1}}{\partial y}(\hat{X}(T), \hat{Y}(T))\left\langle\nabla_{\hat{X}(T)} F, X(T)-\hat{X}(T)\right\rangle\right] \\
&= \mathbb{E}\left\langle\hat{p}_{1}(T), X(T)-\hat{X}(T)\right\rangle \\
&=\mathbb{E}\left[\int_{0}^{T} \hat{p}_{1}(t) d \tilde{X}(t)+\int_{0}^{T} \tilde{X}(t) d \hat{p}_{1}(t)+\int_{0}^{T} \hat{q}_{1}(t) \tilde{\sigma}(t) d t\right],
\end{aligned}
$$


where we have put

$$
\tilde{X}(t):=X(t)-\hat{X}(t), \quad \tilde{\sigma}(t):=\sigma(t)-\hat{\sigma}(t)
$$

Note that

$$
\begin{aligned}
& \mathbb{E}\left[\int_{0}^{T} \hat{p}_{1}(t) d \tilde{X}(t)\right]=\mathbb{E}\left[\int_{0}^{T} \hat{p}_{1}(t)(b(t)-\hat{b}(t)) d t\right. \\
& \left.\quad+\int_{0}^{T} \hat{p}_{1}(t)\left(\lambda_{1} d \xi_{1}(t)-\hat{\lambda}_{1} d \hat{\xi}_{1}(t)+\lambda_{2} d \hat{\xi}_{2}(t)-\hat{\lambda}_{2} d \hat{\xi}_{2}(t)\right)\right] .
\end{aligned}
$$

and that

$$
\begin{aligned}
& \mathbb{E}\left[\int_{0}^{T} \tilde{X}(t) d \hat{p}_{1}(t)\right]=\mathbb{E}\left[\int _ { 0 } ^ { T } \tilde { X } ( t ) \left(\left\{-\frac{\partial H_{1,0}}{\partial x}\left(t, X(t), Y(t), w(t), \hat{p}_{1}(t), \hat{q}_{1}(t), \hat{r}_{1}(t)\right)\right.\right.\right. \\
& \left.\left.\left.\quad-\frac{\partial H_{1,0}}{\partial y}\left(t, X(t), Y(t), w(t), \hat{p}_{1}(t), \hat{q}_{1}(t), \hat{r}_{1}(t)\right) \nabla_{X(t)} F\right\} d t-\sum_{j=1}^{2}\left\{\frac{\partial \lambda_{j}}{\partial x}(t) p_{1}(t)+\frac{\partial h_{1, j}}{\partial x}(t)\right\} d \xi_{j}(t)\right)\right]
\end{aligned}
$$

Combining (4.18) with $I_{3}$ and $I_{4}$ we get

$$
\begin{aligned}
& J_{1}-\hat{J}_{1}:=J_{1}\left(\xi_{1}, u_{1}, \hat{\xi}_{2}, \hat{u}_{2}\right)-J_{1}\left(\hat{\xi}_{1}, \hat{u}_{1}, \hat{\xi}_{2}, \hat{u}_{2}\right) \\
& \leq \mathbb{E}\left[\int_{0}^{T}\left\{H_{1}(t)-\hat{H}_{1}(t)-\frac{\partial \hat{H}_{1}}{\partial x}(X-\hat{X})-\frac{\partial \hat{H}_{1}}{\partial y} \nabla F(X-\hat{X})\right\}\right] \\
& =\mathbb{E}\left[\int_{0}^{T} \mathbb{E}\left[H_{1}(t)-\hat{H}_{1}(t)-\frac{\partial \hat{H}_{1}}{\partial x}(X-\hat{X})-\frac{\partial \hat{H}_{1}}{\partial y} \nabla F(X-\hat{X}) \mid \mathcal{G}_{t}^{(1)}\right]\right]
\end{aligned}
$$

where $\hat{H}_{1}(t)$ means that $H_{1}$ is evaluated at $\left(t, \hat{X}(t), \hat{Y}(t), \hat{\xi}(t), \hat{u}(t), \hat{p}_{1}(t), \hat{q}_{1}(t)\right)$, while $H_{1}(t)$ means that $H_{1}$ is evaluated at $\left(t, X(t), Y(t), \xi_{1}(t), u_{1}(t), \hat{\xi}_{2}(t), \hat{u}_{2}(t), \hat{p}_{1}(t), \hat{q}_{1}(t)\right)$.

Note that by concavity of $H_{1}$ we have

$$
\begin{aligned}
& H_{1}\left(t, X, F(X), \xi_{1}, u_{1}, \hat{\xi}_{2}, \hat{u}_{2}, \hat{p}_{1}, \hat{q}_{1}\right)-H_{1}\left(t, \hat{X}, F(\hat{X}), \hat{\xi}_{1}, \hat{u}_{1}, \hat{\xi}_{2}, \hat{u}_{2}, \hat{p}_{1}, \hat{q}_{1}\right) \\
& \leq \frac{\partial \hat{H}_{1}}{\partial x}(\hat{X})(X-\hat{X})+\frac{\partial \hat{H}_{1}}{\partial y}(\hat{X}) \nabla_{\hat{X}} F(X-\hat{X})+\nabla_{\xi_{1}} \hat{H}_{1}(\hat{\xi})\left(\xi_{1}-\hat{\xi}_{1}\right)+\frac{\partial \hat{H}_{1}}{\partial u_{1}}(\hat{u})\left(u_{1}-\hat{u}_{1}\right)
\end{aligned}
$$

Therefore, to obtain that $J_{1}-\hat{J}_{1} \leq 0$, it suffices that

$$
\mathbb{E}\left[\nabla_{\xi_{1}} \hat{H}_{1}(\hat{\xi}) \mid \mathcal{G}_{t}^{(1)}\right]\left(\xi_{1}-\hat{\xi}_{1}\right) \leq 0 \quad \text { a.s. }
$$

for all $\xi_{1}$, and that

$$
\mathbb{E}\left[\frac{\partial \hat{H}_{1}}{\partial u_{1}}(\hat{u}) \mid \mathcal{G}_{t}^{(1)}\right]\left(u_{1}-\hat{u}_{1}\right) \leq 0 \quad \text { a.s. }
$$


for all $u_{1}$. The inequality (4.22) holds by our assumption (4.10), and the inequality (4.21) holds by our assumption (4.12). The difference

$$
J_{2}\left(\hat{\xi}_{1}, \hat{u}_{1}, \xi_{2}, u_{2}\right)-J_{2}\left(\hat{\xi}_{1}, \hat{u}_{1}, \hat{\xi}_{2}, \hat{u}_{2}\right)
$$

is handled similarly.

We proved above a verification theorem, stating sufficient conditions to obtain a Nash equilibrium. We now establish a partial converse, implying that a Nash equilibrium for the singular control game is a conditional saddle point for the Hamiltonian.

Theorem 4.2 (Necessary maximum principle).

Suppose $\hat{w}_{1}=\left(\hat{\xi}_{1}, \hat{u}_{i}\right) \in \mathcal{A}_{1}$ and $\hat{w}_{2}=\left(\hat{\xi}_{2}, \hat{u}_{2}\right) \in \mathcal{A}_{2}$ constitute a Nash equilibrium for the game, i.e. satisfies (4.2) and (4.3). Then

$$
\mathbb{E}\left[\frac{\partial H_{1}}{\partial u_{1}}\left(t, \hat{X}(t), \hat{Y}(t), \hat{\xi}_{1}(t), u_{1}, \hat{\xi}_{2}(t), \hat{u}_{2}(t), \hat{p}_{1}(t), \hat{q}_{1}(t)\right)_{u_{1}=\hat{u}_{1}(t)} \mid \mathcal{G}_{t}^{(1)}\right]=0 \quad \text { a.s. }
$$

and

$$
\mathbb{E}\left[\frac{\partial H_{2}}{\partial u_{2}}\left(t, \hat{X}(t), \hat{Y}(t), \hat{\xi}_{1}(t), \hat{u}_{1}(t), \hat{\xi}_{2}(t), u_{2}, \hat{p}_{2}(t), \hat{q}_{2}(t)\right)_{u_{2}=\hat{u}_{2}(t)} \mid \mathcal{G}_{t}^{(2)}\right]=0 \quad \text { a.s. }
$$

Moreover, the following variational inequalities hold:

$$
\left\{\begin{array}{l}
\mathbb{E}\left[\frac{\partial H_{i, 0}}{\partial \xi_{i}}(t, \hat{X}(t), \hat{Y}(t), \hat{w}(t))+\lambda_{i}(t, \hat{X}(t), \hat{u}(t)) \hat{p}_{i}(t)+h_{i i}(t, \hat{X}(t), \hat{w}(t)) \mid \mathcal{G}_{t}^{(i)}\right] \leq 0 \\
\text { for all } t, i=1,2, \text { and } \\
\mathbb{E}\left[\frac{\partial H_{i, 0}}{\partial \xi_{i}}(t, \hat{X}(t), \hat{Y}(t), \hat{w}(t))+\lambda_{i}\left(t, \hat{X}(t), \hat{u}(t) \hat{p}_{i}(t)+h_{i i}(t, \hat{X}(t), \hat{w}(t)) \mid \mathcal{G}_{t}^{(i)}\right] d \hat{\xi}_{i}(t)=0\right. \\
\text { for all } t, i=1,2 .
\end{array}\right.
$$

Proof. This theorem can be proved in a way similar to the proof of Theorem 3.4 with an adjustment to the stochastic game case. The adjustment is similar to one in the proof of Theorem 4.1.

\subsection{The zero-sum game case}

In the zero-sum case we have

$$
J_{1}\left(w_{1}, w_{2}\right)+J_{2}\left(w_{1}, w_{2}\right)=0 .
$$

Then the Nash equilibrium $\left(\hat{w}_{1}, \hat{w}_{2}\right) \in \mathcal{A}_{1} \times \mathcal{A}_{2}$ satisfying (4.2)-(4.3) becomes a saddle point for

$$
J\left(w_{1}, w_{2}\right):=J_{1}\left(w_{1}, w_{2}\right)
$$


To see this, note that (4.2)-(4.3) imply that

$$
J_{1}\left(w_{1}, \hat{w}_{2}\right) \leq J_{1}\left(\hat{w}_{1}, \hat{w}_{2}\right)=-J_{2}\left(\hat{w}_{1}, \hat{w}_{2}\right) \leq-J_{2}\left(\hat{w}_{1}, w_{2}\right)
$$

and hence

$$
J\left(w_{1}, \hat{w}_{2}\right) \leq J\left(\hat{w}_{1}, \hat{w}_{2}\right) \leq J\left(\hat{w}_{1}, w_{2}\right) \text { for all } w_{1}, w_{2} .
$$

From this we deduce that

$$
\begin{aligned}
\inf _{w_{2} \in \mathcal{A}_{2}} & \sup _{w_{1} \in \mathcal{A}_{1}} J\left(w_{1}, w_{2}\right) \leq \sup _{w_{1} \in \mathcal{A}_{1}} J\left(w_{1}, \hat{w}_{2}\right) \leq J\left(\hat{w}_{1}, \hat{w}_{2}\right) \\
\leq & \inf _{w_{2} \in \mathcal{A}_{2}} J\left(\hat{w}_{1}, w_{2}\right) \leq \sup _{w_{1} \in \mathcal{A}_{1}} \inf _{w_{2} \in \mathcal{A}_{2}} J\left(w_{1}, w_{2}\right) .
\end{aligned}
$$

Since we always have inf $\sup \geq \sup$ inf, we conclude that

$$
\begin{aligned}
\inf _{w_{2} \in \mathcal{A}_{2}} & \sup _{w_{1} \in \mathcal{A}_{1}} J\left(w_{1}, w_{2}\right)=\sup _{w_{1} \in \mathcal{A}_{1}} J\left(w_{1}, \hat{w}_{2}\right)=J\left(\hat{w}_{1}, \hat{w}_{2}\right) \\
& =\inf _{w_{2} \in \mathcal{A}_{2}} J\left(\hat{w}_{1}, w_{2}\right)=\sup _{w_{1} \in \mathcal{A}_{1}} \inf _{w_{2} \in \mathcal{A}_{2}} J\left(w_{1}, w_{2}\right) .
\end{aligned}
$$

i.e. $\left(\hat{w}_{1}, \hat{w}_{2}\right) \in \mathcal{A}_{1} \times \mathcal{A}_{2}$ is a saddle point for $J\left(w_{1}, w_{2}\right)$.

Hence we want to find $\left(\hat{w}_{1}, \hat{w}_{2}\right) \in \mathcal{A}_{1} \times \mathcal{A}_{2}$ such that

$$
\sup _{w_{1} \in \mathcal{A}_{1}} \inf _{w_{2} \in \mathcal{A}_{2}} J\left(w_{1}, w_{2}\right)=\inf _{w_{2} \in \mathcal{A}_{2}} \sup _{w_{1} \in \mathcal{A}_{1}} J\left(w_{1}, w_{2}\right)=J\left(\hat{w}_{1}, \hat{w}_{2}\right),
$$

where

$$
\begin{aligned}
J\left(w_{1}, w_{2}\right) & =\mathbb{E}\left[\int_{0}^{T} f(t, X(t), Y(t), w(t), \omega) d t+g(X(T), Y(T), \omega)\right. \\
& \left.+\int_{0}^{T} h_{1}(t, X(t), w(t), \omega) d \xi_{1}(t)+\int_{0}^{T} h_{2}(t, X(t), w(t), \omega) d \xi_{2}(t)\right] .
\end{aligned}
$$

As shown in [17], in this case only one Hamiltonian $H$ is needed, namely

$$
\begin{aligned}
& H\left(t, x, y, \xi_{1}, u_{1}, \xi_{2}, u_{2}, p, q\right)\left(d t, d \xi_{1}(t), d \xi_{2}(t)\right) \\
& \quad=H_{0}\left(t, x, y, \xi_{1}, u_{1}, \xi_{2}, u_{2}, p, q\right) d t+\sum_{j=1}^{2}\left\{\lambda_{j}(t, x, u) p+h_{j}(t, x, \xi, u)\right\} d \xi_{j}(t)
\end{aligned}
$$

where

$$
H_{0}(t, x, y, w, p, q):=f(t, x, y, w)+b(t, x, y, w) p+\sigma(t, x, y, w) q
$$

and we have put $g_{i}=g, h_{i}=h_{1, i} ; i=1,2$ and $f_{1}=f=-f_{2}$. 
Moreover, there is only one couple $(p, q)$ of adjoint processes, given by the BSDE

$$
\left\{\begin{aligned}
d p(t) & =-\frac{\partial H_{0}}{\partial x}(t, X(t), Y(t), w(t), p(t), q(t)) d t \\
& \left.-\mathbb{E}\left[\frac{\partial H_{0}}{\partial y}(t, X(t), Y(t), w(t), p(t), q(t))\right] \nabla_{X(t)} F\right) d t \\
& -\sum_{j=1}^{2}\left\{\frac{\partial \lambda_{j}}{\partial x}(t, X(t), u(t)) p(t)+\frac{\partial h_{j}}{\partial x}(t, X(t), w(t))\right\} d \xi_{j}(t)+q(t) d B(t) \\
p(T) \quad= & \frac{\partial g}{\partial x}(X(T), Y(T))+\mathbb{E}\left[\frac{\partial g}{\partial y}(X(T), Y(T))\right] \nabla_{X(T)} F
\end{aligned}\right.
$$

We can now state the corresponding sufficient maximum principle for the zero-sum game:

Theorem 4.3 (Sufficient maximum principle for zero-sum singular mean-field games). Let $\left(\hat{w}_{1}, \hat{w}_{2}\right) \in \mathcal{A}_{1} \times \mathcal{A}_{2}$, with corresponding solutions $\hat{X}(t), \hat{Y}(t), \hat{p}(t), \hat{q}(t)$. Suppose the following holds

- The function

$$
X, w_{1} \rightarrow H\left(t, X, F(X), w_{1}, \hat{w}_{2}(t), \hat{p}(t), \hat{q}(t)\right)
$$

is concave for all t, the function

$$
X, w_{2} \rightarrow H\left(t, X, F(X), \hat{w}_{1}(t), w_{2}, \hat{p}(t), \hat{q}(t)\right)
$$

is convex for all $t$, and the function

$$
X \rightarrow g(X, F(X))
$$

is affine.

- (The conditional maximum property)

$$
\begin{aligned}
& \underset{v_{1} \in A_{1}}{e s s \sup } \mathbb{E}\left[H\left(t, \hat{X}(t), \hat{Y}(t), \hat{\xi}_{1}(t), v_{1}, \hat{\xi}_{2}(t), \hat{u}_{2}(t), \hat{p}(t), \hat{q}(t)\right) \mid \mathcal{G}_{t}^{(1)}\right] \\
& \quad=\mathbb{E}\left[H\left(t, \hat{X}(t), \hat{Y}(t), \hat{\xi}_{1}(t), \hat{u}_{1}(t), \hat{\xi}_{2}(t), \hat{u}_{2}(t), \hat{p}(t), \hat{q}(t)\right) \mid \mathcal{G}_{t}^{(1)}\right]
\end{aligned}
$$

and

$$
\begin{aligned}
& \underset{v_{2} \in A_{2}}{\operatorname{sinf}} \mathbb{E}\left[H\left(t, \hat{X}(t), \hat{Y}(t), \hat{\xi}_{1}(t), \hat{u}_{1}(t), \hat{\xi}_{2}(t), v_{2}, \hat{p}(t), \hat{q}(t)\right) \mid \mathcal{G}_{t}^{(2)}\right] \\
& \quad=\mathbb{E}\left[H\left(t, \hat{X}(t), \hat{Y}(t), \hat{\xi}_{1}(t), \hat{u}_{1}(t), \hat{\xi}_{2}(t), \hat{u}_{2}(t), \hat{p}(t), \hat{q}(t)\right) \mid \mathcal{G}_{t}^{(2)}\right]
\end{aligned}
$$

- (Variational inequalities)

$$
\begin{aligned}
& \underset{\xi_{1}}{e s s} \sup \mathbb{E}\left[H\left(t, \hat{X}(t), \hat{Y}(t), \xi_{1}, \hat{u}_{1}(t), \hat{\xi}_{2}(t), \hat{u}_{2}(t), \hat{p}(t), \hat{q}(t)\right) \mid \mathcal{G}_{t}^{(1)}\right] \\
& \quad=\mathbb{E}\left[H\left(t, \hat{X}(t), \hat{Y}(t), \hat{\xi}_{1}(t), \hat{u}_{1}(t), \hat{\xi}_{2}(t), \hat{u}_{2}(t), \hat{p}(t), \hat{q}(t)\right) \mid \mathcal{G}_{t}^{(1)}\right]
\end{aligned}
$$


and

$$
\begin{aligned}
& \text { essinf } \mathbb{E}\left[H\left(t, \hat{X}(t), \hat{Y}(t), \hat{\xi}_{1}(t), \hat{u}_{1}(t), \xi_{2}, \hat{u}_{2}(t), \hat{p}(t), \hat{q}(t)\right) \mid \mathcal{G}_{t}^{(2)}\right] \\
& \quad=\mathbb{E}\left[H\left(t, \hat{X}(t), \hat{Y}(t), \hat{\xi}_{1}(t), \hat{u}_{1}(t), \hat{\xi}_{2}(t), \hat{u}_{2}(t), \hat{p}(t), \hat{q}(t)\right) \mid \mathcal{G}_{t}^{(2)}\right] .
\end{aligned}
$$

Then $\hat{u}(t)=\left(\hat{u}_{1}(t), \hat{u}_{2}(t)\right)$ is a saddle point for $J\left(u_{1}, u_{2}\right)$.

Proof. The proof is similar to (and simpler than) the proof of Theorem 4.1 and is omitted.

\section{Applications}

\subsection{A mean field investment game}

We return to the mean field singular game of Section 2.2. In this case, we get from (4.4)

$$
\begin{aligned}
& H_{i}\left(t, x, y, \xi_{1}, \xi_{2}, p_{i}, q_{i}\right)\left(d t, d \xi_{1}(t), d \xi_{2}(t)\right) \\
& \quad=\pi \min \left(x, \xi_{1}+\xi_{2}\right)+y b(t) p_{i}+x \sigma(t) q_{i}+h_{1} d \xi_{1}(t)+h_{2} d \xi_{2}(t)
\end{aligned}
$$

and the adjoint equations (4.6) becomes

$$
d p_{i}(t)=-\left[\chi_{\left[0, \xi_{1}+\xi_{2}\right)}(x) \pi(t)+\sigma(t) q_{i}(t)+b(t) p_{i}(t)\right] d t+q_{i}(t) d B(t) ; p_{i}(T)=0 .
$$

The variational inequalities (4.25) get the form

$$
\left\{\begin{array}{l}
\pi(t) \chi_{[0, X(t))}\left(\xi_{1}(t)+\xi_{2}(t)\right)+h_{i}(t) \leq 0 \text { and } \\
{\left[\pi(t) \chi_{[0, X(t))}\left(\xi_{1}(t)+\xi_{2}(t)\right)+h_{i}(t)\right] d \xi_{i}(t)=0 ; \quad i=1,2 .}
\end{array}\right.
$$

and the optimal strategy for factory 1 is:

i) If $\pi(t)+h_{1}(t)<0$, do nothing.

ii) If $\pi(t)+h_{1}(t) \geq 0$ increase $\xi_{1}(t)$ to $X(t)-\xi_{2}^{*}(t)$.

In other words, $\left(\xi_{1}, X\right)$ solves the reflected Skorohod problem

$$
\left\{\begin{array}{l}
\xi_{1}(t) \geq\left(X(t)-\xi_{2}^{*}(t)\right) \chi_{[0, \infty)}\left(\pi(t)+h_{1}(t)\right) \\
{\left[\xi_{1}(t)-\left(X(t)-\xi_{2}^{*}(t)\right) \chi_{[0, \infty)}\left(\pi(t)+h_{1}(t)\right)\right] d \xi_{1}(t)=0 .}
\end{array}\right.
$$

So for given $\xi_{2}^{*}$ we choose $\xi_{1}:=R_{1}\left(\xi_{2}^{*}\right)$ solution of (5.1). Similarly, given $\xi_{1}^{*}$ we choose $\xi_{2}:=R_{2}\left(\xi_{1}^{*}\right)$ as the solution of

$$
\left\{\begin{array}{l}
\xi_{2}(t) \geq\left(X(t)-\xi_{1}^{*}(t)\right) \chi_{[0, \infty)}\left(\pi(t)+h_{2}(t)\right) \\
{\left[\xi_{2}(t)-\left(X(t)-\xi_{1}^{*}(t)\right) \chi_{[0, \infty)}\left(\pi(t)+h_{2}(t)\right)\right] d \xi_{2}(t)=0 .}
\end{array}\right.
$$


Thus, to find the Nash equilibrium we need to solve the following coupled problem:

$$
\left\{\begin{array}{l}
\xi_{1}(t) \geq\left(X(t)-\xi_{2}(t)\right) \chi_{[0, \infty)}\left(\pi(t)+h_{1}(t)\right) \\
{\left[\xi_{1}(t)-\left(X(t)-\xi_{2}(t)\right) \chi_{[0, \infty)}\left(\pi(t)+h_{1}(t)\right)\right] d \xi_{1}(t)=0 ;} \\
\xi_{2}(t) \geq\left(X(t)-\xi_{1}(t)\right) \chi_{[0, \infty)}\left(\pi(t)+h_{2}(t)\right) \\
{\left[\xi_{2}(t)-\left(X(t)-\xi_{1}(t)\right) \chi_{[0, \infty)}\left(\pi(t)+h_{2}(t)\right)\right] d \xi_{2}(t)=0 .}
\end{array}\right.
$$

The above system of reflected Skorohod problems can be solved in the following way:

We divide the interval $[0, T]$ into $0=t_{0}<t_{1}<\cdots<t_{n}=T$ such that on each interval $\left[t_{k}, t_{k+1}\right)$ the signs of $\pi(t)+h_{1}(t)$ and $\pi(t)+h_{2}(t)$ remains unchanged. On each interval $\left[t_{k}, t_{k+1}\right]$, we use the following control principles. If both of the inequalities $\pi(t)+h_{1}(t)<0$ and $\pi(t)+h_{2}(t)<0$ hold, then do nothing. If $\pi(t)+h_{1}(t)<0$ and $\pi(t)+h_{2}(t) \geq 0$, then the first factory does not do anything. The second condition in (5.3) becomes

$$
\left\{\begin{array}{l}
\xi_{2}(t) \geq\left(X(t)-\xi_{1}(t)\right) \\
\left.\left[\xi_{2}(t)-\left(X(t)-\xi_{1}(t)\right)\right)\right] d \xi_{2}(t)=0 .
\end{array}\right.
$$

By Remark 2.7 (namely, Equation (2.8)) of [4],

$$
\xi_{2}(t)=\sup _{t_{k} \leq s \leq t}\left(X(s)-\xi_{1}\left(t_{k}\right)\right)^{+}, \quad t_{k} \leq t \leq t_{k+1} .
$$

Thus, we keep $\xi_{1}(t)=\xi_{1}\left(t_{k}\right)$ unchanged and in the same time increase $\xi_{2}(t)$ to $X(t)-\xi_{1}(t)$. Similar result holds if $\pi(t)+h_{1}(t) \geq 0$ and $\pi(t)+h_{2}(t)<0$.

If both $\pi(t)+h_{1}(t) \geq 0$ and $\pi(t)+h_{2}(t) \geq 0$, then (5.3) becomes

$$
\left\{\begin{array}{l}
\xi_{1}(t) \geq\left(X(t)-\xi_{2}(t)\right) \\
{\left[\xi_{1}(t)-\left(X(t)-\xi_{2}(t)\right)\right] d \xi_{1}(t)=0} \\
\xi_{2}(t) \geq\left(X(t)-\xi_{1}(t)\right) \\
\left.\left[\xi_{2}(t)-\left(X(t)-\xi_{1}(t)\right)\right)\right] d \xi_{2}(t)=0
\end{array}\right.
$$

Let $\xi(t)=\xi_{1}(t)+\xi_{2}(t)$ and then (5.4) is equivalent to

$$
\left\{\begin{array}{l}
\xi(t) \geq X(t) \\
{[\xi(t)-X(t)] d \xi(t)=0 .}
\end{array}\right.
$$

Again by Remark 2.7 (namely, Equation (2.8)) of [4], we have

$$
\xi(t)=\sup _{t_{k} \leq s \leq t} X(s)^{+}, \quad t_{k} \leq t \leq t_{k+1} .
$$

Now we show that any decomposition of $\xi(t)$ into the sum of two nondecreasing processes $\xi_{1}(t)$ and $\xi_{2}(t)$ will solve (5.4). In fact, assume $\xi(t)=\xi_{1}(t)+\xi_{2}(t)$, where $\xi_{1}$ and $\xi_{2}$ are two nondecreasing processes. Since $\xi(t) \geq X(t)$ and $\xi_{1}$ and $\xi_{2}$ are nondecreasing, we have

$$
\left\{\begin{array}{l}
{[\xi(t)-X(t)] d \xi_{1}(t) \geq 0} \\
{[\xi(t)-X(t)] d \xi_{2}(t) \geq 0}
\end{array}\right.
$$


Adding them we have

$$
[\xi(t)-X(t)] d \xi_{1}(t)+[\xi(t)-X(t)] d \xi_{2}(t)=[\xi(t)-X(t)] d \xi(t)=0 .
$$

This implies

$$
\left\{\begin{array}{l}
{[\xi(t)-X(t)] d \xi_{1}(t)=0} \\
{[\xi(t)-X(t)] d \xi_{2}(t)=0}
\end{array}\right.
$$

Thus, $\xi_{1}$ and $\xi_{2}$ satisfies (5.4). Summarizing we have

Proposition 5.1. Assume that we can divide the interval $[0, T]$ into $0=t_{0}<t_{1}<\cdots<$ $t_{n}=T$ such that on each interval $\left[t_{k}, t_{k+1}\right)$ the signs of $\pi(t)+h_{1}(t)$ and $\pi(t)+h_{2}(t)$ remain unchanged. Then we can recursively find the solution $\xi_{1}$ and $\xi_{2}$ on each interval $\left[t_{k}, t_{k+1}\right]$ for $k=0,1, \cdots, n-1$. On the interval $\left[t_{k}, t_{k+1}\right]$, we have

(i) If both inequalities $\pi(t)+h_{1}(t)<0$ and $\pi(t)+h_{2}(t)<0$ hold, then do nothing.

(ii) If $\pi(t)+h_{1}(t)<0$ but $\pi(t)+h_{2}(t) \geq 0$, then

$$
\xi_{1}(t)=\xi_{1}\left(t_{k}\right), \quad t_{k} \leq t \leq t_{k+1}
$$

and

$$
\xi_{2}(t)=\sup _{t_{k} \leq s \leq t}\left(X(s)-\xi_{1}\left(t_{k}\right)\right)^{+}, \quad t_{k} \leq t \leq t_{k+1} .
$$

If $\pi(t)+h_{1}(t) \geq 0$ but $\pi(t)+h_{2}(t)<0$, then

$$
\xi_{2}(t)=\xi_{2}\left(t_{k}\right), \quad t_{k} \leq t \leq t_{k+1}
$$

and

$$
\xi_{1}(t)=\sup _{t_{k} \leq s \leq t}\left(X(s)-\xi_{2}\left(t_{k}\right)\right)^{+}, \quad t_{k} \leq t \leq t_{k+1} .
$$

(iii) If both inequalities $\pi(t)+h_{1}(t) \geq 0$ and $\pi(t)+h_{2}(t) \geq 0$ hold, then $\xi_{1}$ and $\xi_{2}$ can be any nondecreasing processes such that

$$
\xi_{1}(t)+\xi_{2}(t)=\sup _{t_{k} \leq s \leq t} X(s)^{+}, \quad t_{k} \leq t \leq t_{k+1} .
$$

Note that in this case the Nash equilibrium is not unique.

\subsection{Model uncertainty singular control}

We represent model uncertainty by a family of probability measures $Q=Q^{\theta}$ equivalent to $P$, with the Radon-Nikodym derivative on $\mathcal{F}_{t}$ given by

$$
\frac{d\left(Q \mid \mathcal{F}_{t}\right)}{d\left(P \mid \mathcal{F}_{t}\right)}=G^{\theta}(t)
$$


where, for $0 \leq t \leq T, G^{\theta}(t)$ is an exponential martingale of the form

$$
d G^{\theta}(t)=G^{\theta}(t) \theta(t) d B(t) ; \quad G^{\theta}(0)=1 .
$$

Here $\theta$ may be regarded as a scenario control. Let $\mathcal{A}_{1}$ denote a given family of admissible singular controls $\xi$ and let $\mathcal{A}_{2}$ denote a given set of admissible scenario controls $\theta$ such that

$$
\mathbb{E}\left[\int_{0}^{T} \theta^{2}(t) d t\right]<\infty .
$$

Now assume that $X_{1}(t)=X_{1}^{\xi}(t)$ is a singularly controlled mean-field Itô process of the form

$$
d X_{1}(t)=b_{1}\left(t, X_{1}(t), Y(t), \omega\right) d t+\sigma_{1}\left(t, X_{1}(t), Y(t), \omega\right) d B(t)+\lambda_{1}\left(t, X_{1}(t), \omega\right) d \xi(t),
$$

where

$$
Y(t)=F\left(X_{1}(t, \cdot)\right)
$$

and $F$ is a Fréchet differentiable operator on $L^{2}(P)$. Let $\mathbb{G}^{(1)}=\left\{\mathcal{G}_{t}^{(1)}\right\}_{0 \leq t \leq T}$ and $\mathbb{G}^{(2)}=$ $\left\{\mathcal{G}_{t}^{(2)}\right\}_{0 \leq t \leq T}$ be given subfiltrations of $\mathbb{F}=\left\{\mathcal{F}_{t}\right\}_{0 \leq t \leq T}$, representing the information available to the controllers at time $t$. It is required that $\xi \in \mathcal{A}_{1}$ be $\mathbb{G}^{(1)}$-predictable, and $\theta \in \mathcal{A}_{2}$ be $\mathbb{G}^{(2)}$-predictable. We consider the stochastic differential game to find $(\hat{\xi}, \hat{\theta}) \in \mathcal{A}_{1} \times \mathcal{A}_{2}$ such that

$$
\sup _{\xi \in \mathcal{A}_{1}} \inf _{\theta \in \mathcal{A}_{2}} \mathbb{E}_{Q^{\theta}}[j(\xi, \theta)]=\mathbb{E}_{Q^{\hat{\theta}}}[j(\hat{\xi}, \hat{\theta})]=\inf _{\theta \in \mathcal{A}_{2}} \sup _{\xi \in \mathcal{A}_{1}} \mathbb{E}_{Q^{\theta}}[j(\xi, \theta)],
$$

where

$$
\begin{aligned}
j(\xi, \theta)= & \int_{0}^{T}\left\{f_{1}\left(t, X_{1}(t), Y(t), \xi(t), \omega\right)+\rho(\theta(t))\right\} d t \\
& +g_{1}\left(X_{1}(T), Y(T), \omega\right)+\int_{0}^{T} h_{1}\left(t, X_{1}(t), \omega\right) \xi(d t) .
\end{aligned}
$$

The term $\mathbb{E}_{Q^{\theta}}\left[\int_{0}^{T} \rho(\theta(t)) d t\right]$ can be seen as a penalty term, penalizing the difference between $Q^{\theta}$ and the original probability measure $P$. Note that since $G^{\theta}(t)$ is a martingale we have

$$
\begin{aligned}
\mathbb{E}_{Q^{\theta}}[j(\xi, \theta)] & =\mathbb{E}\left[\int_{0}^{T} G^{\theta}(t)\left\{f_{1}\left(t, X_{1}(t), Y(t), \xi(t)\right)\right)+\rho(\theta(t)\} d t+G^{\theta}(T) g_{1}\left(X_{1}(T), Y(T)\right)\right. \\
& \left.+\int_{0}^{T} G^{\theta}(t) h_{1}\left(t, X_{1}(t)\right) d \xi(t)\right]=: J(\xi, \theta) .
\end{aligned}
$$

We see that this is a mean-field singular control stochastic differential game of the type discussed in Section 4.2, with a two-dimensional state space $X(t):=\left(X_{1}(t), X_{2}(t)\right)$, with $X_{2}(t)=G^{\theta}(t)$ and with

$$
\begin{aligned}
f(t, X(t), Y(t), \xi(t), \theta(t))) & :=X_{2}(t)\left\{f_{1}\left(t, X_{1}(t), Y(t), \xi(t)\right)+\rho(\theta(t))\right\}, \\
g(X(T), Y(T)) & :=X_{2}(T) g_{1}\left(X_{1}(T), Y(T)\right), \\
h(t, X(t)) & :=X_{2}(t) h_{1}\left(t, X_{1}(t)\right) .
\end{aligned}
$$


We get the following Hamiltonian for the game (5.10):

$$
\begin{aligned}
& H\left(t, x_{1}, x_{2}, y, \xi, \theta, p, q, r\right)(d t, d \xi(t)) \\
& \quad=H_{0}\left(t, x_{1}, x_{2}, y, \xi, \theta, p, q\right) d t+\left\{\lambda_{1}\left(t, x_{1}\right) p_{1}+x_{2} h_{1}\left(t, x_{1}\right)\right\} d \xi(t)
\end{aligned}
$$

where

$$
H_{0}\left(t, x_{1}, x_{2}, y, \xi, \theta, p, q\right)=x_{2}\left\{f_{1}\left(t, x_{1}, y, \xi\right)+\rho(\theta)\right\}+b_{1}\left(t, x_{1}, y\right) p_{1}+\sigma\left(t, x_{1}, y\right) q_{1}+x_{2} \theta q_{2} .
$$

The corresponding mean-field BSDEs for the adjoint processes become

$$
\left\{\begin{aligned}
d p_{1}(t) & =-\frac{\partial H_{0}}{\partial x_{1}}(t, X(t), Y(t), \xi(t), \theta(t), p(t), q(t)) d t \\
& \left.-\mathbb{E}\left[\frac{\partial H_{0}}{\partial y}(t, X(t), Y(t), \xi(t), \theta(t), p(t), q(t))\right] \nabla_{X_{1}(t)} F\right) d t \\
& -\left\{\frac{\partial \lambda_{1}}{\partial x_{1}}\left(t, X_{1}(t)\right) p_{1}(t)+x_{2} \frac{\partial h_{1}}{\partial x_{1}}\left(t, X_{1}(t)\right)\right\} d \xi(t)+q_{1}(t) d B(t) \\
p_{1}(T) & =X_{2}(T) \frac{\partial g_{1}}{\partial x_{1}}\left(X_{1}(T), Y(T)\right)+\mathbb{E}\left[\frac{\partial g_{1}}{\partial y}\left(X_{1}(T), Y(T)\right)\right] \nabla_{X_{1}(T)} F
\end{aligned}\right.
$$

and

$$
\left\{\begin{array}{l}
d p_{2}(t)=-\left\{f_{1}\left(t, X_{1}(t), Y(t), \xi(t)\right)+\rho(\theta(t))+\theta(t) q_{2}(t)\right\} d t+q_{2}(t) d B(t) \\
p_{2}(T)=g_{1}\left(X_{1}(T), Y(T)\right)
\end{array}\right.
$$

Minimizing the Hamiltonian with respect to $\theta$ gives the following first order condition:

$$
\frac{\partial \rho}{\partial \theta}(t)=-\mathbb{E}\left[q_{2}(t) \mid \mathcal{G}_{t}^{(2)}\right]
$$

The variational inequalities $(4.25)$ reduce to

$$
\left\{\begin{array}{l}
\mathbb{E}\left[\hat{X}_{2}(t) \nabla_{\xi} f_{1}\left(t, \hat{X}_{1}(t), \hat{Y}(t), \hat{\xi}(t), \hat{\theta}(t)\right)+\lambda_{1}\left(t, \hat{X}_{1}(t)\right) \hat{p}_{1}(t)+\hat{X}_{2}(t) h_{1}\left(t, \hat{X}_{1}(t)\right) \mid \mathcal{G}_{t}^{(1)}\right] \leq 0 \\
\mathbb{E}\left[\hat{X}_{2}(t) \nabla_{\xi} f_{1}\left(t, \hat{X}_{1}(t), \hat{Y}(t), \hat{\xi}(t), \hat{\theta}(t)\right)+\lambda_{1}\left(t, \hat{X}_{1}(t)\right) \hat{p}_{1}(t)+\hat{X}_{2}(t) h_{1}\left(t, \hat{X}_{1}(t)\right) \mid \mathcal{G}_{t}^{(1)}\right] d \hat{\xi}(t)=0 .
\end{array}\right.
$$

In general it seems to be a formidable mathematical challenge to solve such a coupled system of forward-backward singular SDEs. However, in some cases a possible solution procedure could be described, as in the next example.

\subsection{Optimal harvesting under uncertainty}

Let us consider a model uncertainty version of the optimal harvesting problem introduced in Section 2.1. For simplicity we put $K=1$. Thus we have the following mean-field forward system $\left(X^{\xi}, G^{\theta}\right)$, where $G^{\theta}$ is given by (5.6) and

$$
d X(t)=d X^{\xi}(t)=\mathbb{E}\left[X^{\xi}(t)\right] b(t) d t+X^{\xi}(t) \sigma(t) d B(t)-\lambda_{0}(t) d \xi(t) ; X^{\xi}\left(0^{-}\right)=x>0,
$$


with performance functional

$$
J(\xi, \theta)=\mathbb{E}\left[\int_{0}^{T} G^{\theta}(t) \rho(\theta(t)) d t+\int_{0}^{T} G^{\theta}(t) h_{0}(t) X^{\xi}(t) d \xi(t)+G^{\theta}(T) X^{\xi}(T)\right] .
$$

The model uncertainty harvesting problem is to find $\left(\xi^{*}, \theta^{*}\right) \in \mathcal{A}_{1} \times \mathcal{A}_{2}$ such that

$$
\sup _{\xi \in \mathcal{A}_{1}} \inf _{\theta \in \mathcal{A}_{2}} J\left(\xi, \theta=\inf _{\theta \in \mathcal{A}_{2}} \sup _{\xi \in \mathcal{A}_{1}} J(\xi, \theta)=J\left(\xi^{*}, \theta^{*}\right)\right.
$$

We assume that the controllers have full information, so it is required that $\xi \in \mathcal{A}_{1}$ be $\mathbb{F}$-predictable, and $\theta \in \mathcal{A}_{2}$ be $\mathbb{F}$-predictable.

Here the Hamiltonian is

$$
\begin{aligned}
& H(t, x, g, y, \xi, \theta, p, q)(d t, \xi(d t)) \\
& =\left\{g \rho(\theta)+y b(t) p_{1}+x \sigma(t) q_{1}+g \theta q_{2}\right\} d t+\left\{-\lambda_{0}(t) p_{1}+x g h_{0}(t)\right\} d \xi(t) .
\end{aligned}
$$

Minimizing the Hamiltonian with respect to $\theta$ gives the first order equation

$$
\rho^{\prime}(\theta)(t)=-q_{2}(t)
$$

The corresponding reflected backward system is

$$
\begin{aligned}
& d p_{1}(t)=-\left[b(t) p_{1}(t)+\sigma(t) q_{1}(t)\right] d t-h_{0}(t) G^{\theta}(t) d \xi(t)+q_{1}(t) d B(t) ; p_{1}(T)=G^{\theta}(T) \\
& d p_{2}(t)=-\left[\rho(\theta(t))+\theta(t) q_{2}(t)\right] d t-h_{0}(t) X(t) d \xi(t)+q_{2}(t) d B(t) ; \quad p_{2}(T)=X(T)
\end{aligned}
$$

with variational inequalities

$$
\left\{\begin{array}{l}
-\lambda_{0}(t) p_{1}(t)+X(t) G^{\theta}(t) h_{0}(t) \leq 0 \\
{\left[-\lambda_{0}(t) p_{1}(t)+X(t) G^{\theta}(t) h_{0}(t)\right] d \xi(t)=0}
\end{array}\right.
$$

Then we get the following result:

Proposition 5.2. Suppose there exists a solution $\hat{X}(t):=X^{\hat{\xi}}(t), \hat{G}(t):=G^{\hat{\theta}}(t), \hat{p}_{1}(t)$, $\hat{q}_{1}(t), \hat{p}_{2}(t), \hat{q}_{2}(t), \hat{\xi}(t), \hat{\theta}(t)$ of the coupled system of mean-field forward-backward singular stochastic differential equations consisting of the forward equations (5.22) and the reflected backward equations (5.27),(5.28), and satisfying the constraint (5.29). Then $\hat{\xi}(t)$ is the optimal harvesting strategy and $\hat{\theta}(t)$ is the optimal scenario parameter for the model uncertainty harvesting problem (5.24).

\section{References}

[1] Anderson, D. and Djehiche, B. A maximum principle for SDEs of mean-field type. Applied Mathematics and Optimization 63, 341-356 (2011). 
[2] Bensoussan, B .; Frehse, J. and Yam, P. Mean Field Games and Mean Field Type control Theory, Springer Briefs in Mathematics, 2013.

[3] Buckdahn, R.; Djehiche, B. and Li, J. A general stochastic maximum principle for SDEs of mean-field type, Applied Mathematics and Optimization 64 (2011), no. 2, 197-216.

[4] Burdzy, K.; Kang, W. and Ramanan, K. The Skorokhod problem in a time-dependent interval. Stochastic Process. Appl. 119 (2009), no. 2, 428-452.

[5] Carmona, R. and Delarue, F. Forward-backward stochastic differential equations and controlled McKean Vlasov dynamics, arXiv preprint arXiv:1303.5835 (2013).

[6] Carmona, R.; Delarue, F. and Lachapelle, A. Control of McKean-Vlasov dynamics versus mean field games, Mathematics and Financial Economics 7 (2013), no. 2, 131-166.

[7] Dahl, K. R. and Øksendal, B., Singular recursive utility. arXiv:1244132 (April 2015) To appear in Stochastics.

[8] M. Freidlin, Functional Integration and Partial Differential Equations, Princeton University Press, Princeton NJ, USA, 1985.

[9] Hamadène, S. Backward-forward SDE's and stochastic differential games. Stochastic Processes and their Applications 77, 1-15 (1998).

[10] Hosking, J. J. A. A stochastic maximum principle for a stochastic differential game of mean-field type. Applied Mathematics and Optimization, 66(3), 415-454, (2012).

[11] Hu, Y.; Nualart, D. and Song, X. Malliavin calculus for backward stochastic differential equations and application to numerical solutions. Ann. Appl. Probab. 21 (2011), 23792423.

[12] Huang, M.; Malhamé, R. and Caines, P. Large population stochastic dynamic games: closed- loop McKean-Vlasov systems and the Nash certainty equivalence principle, Communications in Information and Systems 6 (2006), no. 3, 221-252.

[13] Kobila, Т. Ø. A class of solvable stochastic investment problems involving stochastic maximum principle for a stochastic differential game of mean-field type. Stochastics and Stochastics Reports 43, 29-63 (1993).

[14] Lasry J. M. and Lions, P. L. Mean field games, Japanese Journal of Mathematics 2 (2007), 229-260.

[15] Meyer-Brandis, T.; Øksendal, B. and Zhou, X.Y. A mean-field stochastic maximum principle via Malliavin calculus, Stochastics 84 (2012), 643-666.

[16] Øksendal, B. and Sulem, A. Singular control and optimal stopping with partial information of jump diffusions. SIAM J. Control Optimization 50 (2012), 2254-2287. 
[17] Øksendal, B. and Sulem, A. Forward-backward stochastic differential games and stochastic control under model uncertainty. J. Optim. Theory Appl. 161 (2012), 22-55. DOI 10.1007/s10957-012-0166-7.

[18] Øksendal, B. and Sulem, A. Risk minimization in financial markets modeled by Itô-Lévy processes, Afrika Matematika 26 (2015), 939-979. DOI:10.1007/s13370-014-0248-9

[19] Øksendal, B., Sulem, A. and Zhang, T.: Singular control and optimal stopping of SPDEs, and backward SPDEs with reflection. Math. Oper. Research 39 (2014), 464-486.

[20] Pindyck, R.S. Irreversible investments, capacity choice, and the value of the firm. American Economic Review 78 (1988), 969-985.

[21] Pindyck, R.S. Irreversibility and the explanation of investment behaviour. In D. Lund and B. Øksendal (editors) Stochastic Models and Option Values. North-Holland 1991.

[22] Pindyck, R.S. Irreversibility, uncertainty and investment. J. Economic Literature 29 (1991), 110-1148.

[23] Peng, S. G. A general stochastic maximum principle for optimal control problems. SIAM J. Control Optim. 28 (1990), no. 4, 966-979.

[24] Prévôt, C. and Roeckner, M.: A concise course on stochastic partial differential equations. Lecture Notes in Math. 1905. Springer 2007.

[25] Yong, J.; Zhou, X. Stochastic controls. Hamiltonian systems and HJB equations. Applications of Mathematics (New York), 43. Springer-Verlag, New York, 1999.

[26] Zhang, L. The relaxed stochastic maximum principle in the mean field singular controls. arXiv 1202.4129v5, 1 Nov 2012. 